\title{
The vertical structure of T Tauri accretion discs
}

\section{Consistent interpretation of spectra and visibilities with a two-layer model}

\author{
R. Lachaume ${ }^{1}$, F. Malbet ${ }^{1}$, and J.-L. Monin ${ }^{1,2}$ \\ ${ }^{1}$ Laboratoire d'Astrophysique UMR UJF-CNRS 5571, Observatoire de Grenoble, Université Joseph Fourier, BP 53, \\ 38041 Grenoble Cedex 9, France \\ 2 Institut Universitaire de France
}

Received 27 May 2002 / Accepted 10 December 2002

\begin{abstract}
We present a two-layer accretion disc model developed to simultaneously fit optical long baseline visibilities and spectral energy distributions of T Tauri accretion discs. This model allows us to access easily the physical conditions in the disc as the mid-plane or the surface temperature.

Our model includes viscous heating, absorption of stellar irradiation, and thermalisation with the surrounding medium. The disc is modelled with concentric cylinders for which the vertical radiation transfer is computed using two layers with vertically averaged temperatures: the outer layer is heated by the stellar irradiation and by the inner layer, and the inner layer by viscous dissipation and by the outer layer. We investigate three prescriptions for the geometrical thickness of the disc: it is either proportional the scale height (model 1), given ad hoc (model 2), or zero (model 3). We then derive the disc structure in the case of the $\alpha$ and $\beta$ viscosity prescriptions, as well as for various optical thickness regimes of the disc.

This analytical model allows us to disentangle regions where the mid-plane temperature and the effective temperature are dominated by accretion from regions dominated by reprocessing of stellar light. In the case of $\alpha$-prescription, we find that the structure of model 2 gives predictions very close to those of numerical simulations from previous authors.

From the disc structure, we derive the spectral energy distributions, images and interferometric visibilities. We analyse the influence of the disc parameters on the resulting structure and on the observable outputs. We apply our model to interpret consistently the spectral energy distributions and visibilities of SU Aur and FU Ori for which interferometric data are available, and that are not known to be part of a multiple system. We were not able to derive a consistent fit for T Tau North, which might come from caveats in the flux correction from its South component, but were able to separately derive fits for its spectrum and its visibilities.

We find that even a single interferometric measurement at one infrared wavelength can bring a very strong constraint on disc models. We predict that future massive interferometric observations of accretion discs will provide a breakthrough in the understanding of accretion disc physics.
\end{abstract}

Key words. stars: pre-main sequence - stars: circumstellar matter - accretion, accretion disks - methods: analytical methods: numerical - methods: data analysis

\section{Introduction}

Since the initial models of viscous accretion discs by Shakura \& Sunyaev (1973) and Lynden-Bell \& Pringle (1974), the physics of the close environment of T Tauri stars (TTS) has been extensively studied in order to interpret their spectral energy distribution (SED). For the sake of simplicity, models traditionally separated discs into two categories, sometimes called active discs, on one hand, in which viscous dissipation is predominant, and passive discs, on the other hand, for which irradiation by the central star is the main heating process. Early models used quasi-Keplerian steady accretion discs, assumed

Send offprint requests to: $\mathrm{R}$. Lachaume,

e-mail: Regis.Lachaume@obs.ujf-grenoble.fr to be geometrically thin for a wide range of accretion rate; they predicted a fixed slope for the infrared spectrum: $\lambda F_{\lambda} \sim \lambda^{-4 / 3}$. However many TTS present flatter SEDs, and disc flaring was among the first attempts to explore disc vertical structure as an explanation for such SED flattening (Adams et al. 1987; Kenyon \& Hartmann 1987): in a flared disc, the surface of the remote parts is tilted toward the star and gets more stellar light than forecast by the standard model, resulting in a warmer disc further away from the star. Since then, several models have been proposed in order to explain both standard SEDs and flatter ones.

An analytical study of the radiative transfer in the vertical structure of discs was first carried out by Hubeny (1990) for active discs, then by Malbet \& Bertout (1991, hereafter 
Paper I) for passive discs. In the latter model, the topmost layers of the disc, illuminated by the star, are hotter than the disc photosphere, resulting in excess continuum and line emission. Later on, Chiang \& Goldreich $(1997,1999)$ used a simplified two-layer passive disc model based on the same superheating mechanism and derived SEDs, confirming conclusions of Paper I and producing results consistent with observations. More recently, Malbet et al. (2001, hereafter Paper II) generalised the analytical study of Paper I to discs heated by several processes, and used its formalism to derive the the vertical structure of active discs.

On the other hand, numerical integration of the equations of radiative transfer was carried out by various authors in order to derive the vertical structure of accretion discs with fewer a priori approximations. Bell \& Lin (1994); Bell et al. (1997) developed an active disc model in order to explain FU Orionis outbursts; D'Alessio et al. $(1998,1999)$ dealt with the more general case of a disc heated both by viscous heating and stellar irradiation.

From a general point of view, all these studies predict spectra consistent with observations, but they have rarely been checked consistently against the spatial information revealed by recent optical and infrared high angular resolution imaging. Recently, the advent of optical interferometry has set newer constraints on disc models. Malbet et al. (1998); Akeson et al. (2000, 2002); Malbet \& Berger (2002) obtained the first visibility measurements of TTS and FU Orionis stars. However, they failed to consistently fit both SEDs and visibilities with a standard disc model: most of the time, the disc parameters derived from the SED data are in disagreement with those derived from visibility data. No other attempt to compare self-consistent disc models and interferometric measurements has been carried out so far for low mass pre-main sequence stars.

In this paper, we tackle the issue of analytically describing a disc in presence of the two main heating processes, viscous heating and stellar irradiation, the latter requiring a correct description of the flaring. This model suits the TTS and FU Ori-type stars for which viscous heating cannot be ignored; for more massive stars (Herbig $\mathrm{Ae} / \mathrm{Be}$ ) it has been shown (Dullemond et al. 2001) that viscosity, as a heating mechanism, can be ignored, so that our model is not relevant. In Sect. 2, we present a two-layer version of the model developed in Paper I and carry out an analytical determination of the structure of the disc. We derive a set of equations giving the mid-plane temperature and the flaring index, from which the whole structure can be determined. In Sect. 3, we briefly present the numerical approach. In Sect. 4, we compare the results of the model with other models and analyse the influence of some disc parameters on the observables. In Sect. 5, we apply our model to the few low-mass young stellar objects (YSOs) observed in optical interferometry.

\section{Model description}

\subsection{The two-layer structure}

The standard accretion disc model by Shakura \& Sunyaev (1973) determines the disc emission with its effective

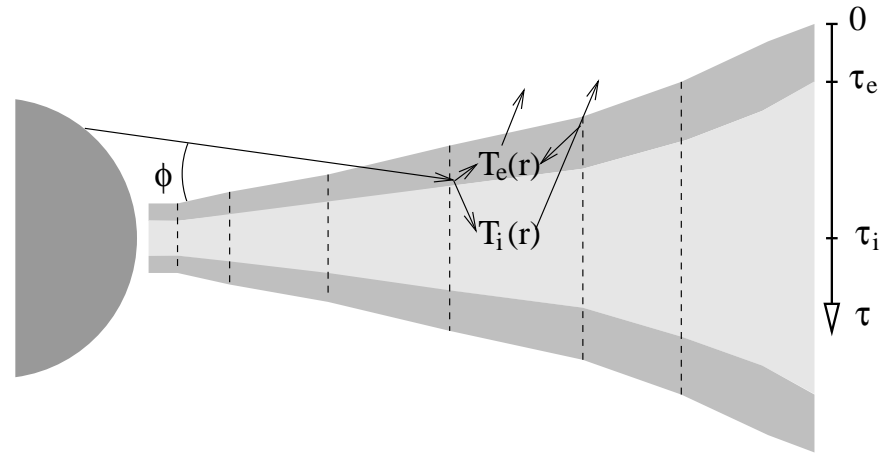

Fig. 1. Radial slice of a two-layer disc.

temperature and does not take the vertical temperature profile into account. However, this approximation fails when the disc surface is super-heated by stellar irradiation (Paper I): the illumination by the star is predominant in a optically thin outer layer of the disc while the optically thick part of the disc is governed by the radiative transfer of thermal radiation. This phenomenon results in emission at different temperatures and has a strong incidence on the SED.

To further explore the resulting structure of the disc, we use a two-layer vertical structure. This approach was first proposed by Chiang \& Goldreich (1997) in the case of passive discs, and we apply it here in a more general case where viscous dissipation, stellar irradiation, and thermalization with the surrounding medium are taken into account. In the case of a passive disc the disc physical properties variations with distance to the star follow a unique power law (Chiang \& Goldreich 1997). In an active disc, where the temperature is dominated by viscous dissipation, this is no longer the case (Paper II, see Fig. 3), due to the sensitivity of the vertical structure to the disc material opacity.

In this paper, we present a model of disc that can be seen as a simplification of the formalism of Paper II. In this model, we consider an optically thick disc heated both by stellar irradiation and viscous dissipation. An optically and geometrically thin outer layer is directly heated by the star and by the inner layer; the optically thick inner layer is heated by viscous dissipation and by the outer layer. In the outer layer we use a vertically averaged temperature to solve the radiative transfer, and in the inner layer we use the mid-plane temperature. A radial slice of such a disc is represented in Fig. 1. The inner and outer layers have the optical thicknesses $\tau_{\mathrm{i}}$ and $\tau_{\mathrm{e}}$, and the temperatures $T_{\mathrm{i}}$ and $T_{\mathrm{e}}$ respectively.

The incidence of stellar radiation onto the disc is given by the angle $\phi$, which is an average of this incidence over the surface of the star. The height of the outer layer is $\mathcal{H}$.

In this section, we focus our attention on two quantities: the mid-plane temperature and the flaring angle. The structure of the disc can be derived from these two quantities; for instance, the mid-plane temperature governs the scale height and the flaring angle the surface temperature. In order to obtain $T_{\mathrm{i}}$ and $\gamma$ we proceed in four steps: in Sect. 2.2, we derive the effective temperatures $T_{\mathrm{v}}, T_{\mathrm{r}}$ and $T_{\mathrm{a}}$ corresponding to viscous heating, stellar light reprocessing and thermalization with the disc surrounding medium. In Sect. 2.3, equations of transfer 
are obtained, giving the temperatures $T_{\mathrm{i}}$ and $T_{\mathrm{e}}$ as a function of the optical depths $\tau_{\mathrm{i}}$ and $\tau_{\mathrm{e}}$ and the effective temperatures $T_{\mathrm{v}}$, $T_{\mathrm{r}}$ and $T_{\mathrm{a}}$. In Sect. 2.4, the structure of the disc (scale height, column density, optical depth, etc.) is connected to the temperatures $T_{\mathrm{i}}$ and $T_{\mathrm{e}}$. In Sect. 2.5, the results from the first three steps are combined to derive the mid-plane temperature $T_{\mathrm{i}}$ and the flaring (variation of $\mathcal{H} / r$ with $r$ ) in a set of coupled equations.

\subsection{Heating sources}

Lynden-Bell \& Pringle (1974) showed that the effective temperature of a geometrically thin active disc is

$T_{\mathrm{v}}^{4}=\frac{3}{8} \frac{\Omega_{\mathrm{K}}^{2} \dot{M} f}{\sigma \pi}$

where $f$ is given by

$f=1-\sqrt{\frac{r_{*}}{r}}$.

$\dot{M}, \Omega_{\mathrm{K}}$ and $r_{*}$ are respectively the accretion rate, the Keplerian angular velocity at radius $r$, and the radius of the star.

We suppose that the surface of the disc presents a mean albedo $\mathcal{A}$ constant over the whole disc. The effective temperature associated with stellar heating then reads

$T_{\mathrm{r}}^{4} \approx \phi T_{\mathrm{eq}}^{4}$.

$T_{\text {eq }}$ is the Local Thermal Equilibrium temperature of a body with an albedo $\mathcal{A}$ at a distance $r$ of the star. It is given by

$T_{\mathrm{eq}}^{4}=(1-\mathcal{A}) \frac{T_{*}^{4}}{2}\left(\frac{r_{*}}{r}\right)^{2}$,

where $T_{*}$ is the effective temperature of the star.

\subsection{Equations of transfer}

Since the layers are assumed to be isothermal, the radiative transfer has a simple form, depending only on the optical depths of the layers and their temperatures.

If viscous dissipation only occurs, we expect a surface temperature of

$T_{\mathrm{e}}^{4}=\frac{T_{\mathrm{v}}^{4}}{2}$

as in a stellar atmosphere within the Eddington approximation. Concerning the temperature in the mid-plane of the disc, as showed in Paper II, the stellar atmosphere formalism cannot be used as is. A corrective term $\Delta \tau_{\mathrm{i}}$ must be applied to the optical thickness $\tau_{\mathrm{i}}$ :

$T_{\mathrm{i}}^{4}=\frac{3}{4} T_{\mathrm{v}}^{4}\left(\tau_{\mathrm{i}}-\Delta \tau_{\mathrm{i}}\right)$.

For the sake of simplicity we ignored the usual constant term $2 / 3$ before the large optical depth.

If reprocessing only occurs, the outer layer is super-heated because it is optically thin: the stellar irradiation is dissipated along a slanted path of optical depth unity in the visible, i.e. much less than one for the reprocessed IR radiation in the vertical direction. We follow Chiang \& Goldreich (1997) and state that both layers present a vertically averaged temperature. The outer layer catches the flux $T_{\mathrm{r}}^{4}$ and emits half of it upward, while being optically thin, so that

$T_{\mathrm{e}}^{4}=\frac{T_{\mathrm{r}}^{4}}{2 \tau_{\mathrm{e}}}$

and emits the other half down to the optically thick inner layer, hence

$T_{\mathrm{i}}^{4}=\frac{T_{\mathrm{r}}^{4}}{2}$.

In the case of thermalization with the disc surrounding medium, the temperatures are

$T_{\mathrm{i}}^{4}=T_{\mathrm{a}}^{4}$,

$T_{\mathrm{e}}^{4}=T_{\mathrm{a}}^{4}$.

In the general case, the three heating contributions considered above occur together. The superposition principle can be applied to the specific radiative intensities; that is the radiation at a given location of the disc is the sum of the radiations corresponding to different heating processes (see Paper II). Summing over Eqs. (5)-(7) we derive

$T_{\mathrm{i}}^{4}=T_{\mathrm{a}}^{4}+\frac{T_{\mathrm{r}}^{4}}{2}+\frac{3}{4} T_{\mathrm{v}}^{4}\left(\tau_{\mathrm{i}}-\Delta \tau_{\mathrm{i}}\right)$

$T_{\mathrm{e}}^{4}=T_{\mathrm{a}}^{4}+\frac{T_{\mathrm{r}}^{4}}{2 \tau_{\mathrm{e}}}+\frac{T_{\mathrm{v}}^{4}}{2}$

This result can also be obtained from the equations of transfer 28-31 of Paper II using the following approximations: (i) the surface is optically thin, (ii) both layers are isothermal, (iii) viscosity only occurs in the inner layer, (iv) diffusion is ignored, (v) no stellar radiation penetrates the inner layer, and (vi) the vertical optical thickness of the disc is larger than unity.

\subsection{Structure of the disc}

If we ignore self-gravity, the vertical gravitational field is proportional to the distance $z$ from the mid-plane. Since the inner layer is isothermal, the density reads

$\rho(z)=\rho_{\mathrm{i}} \exp \left(-\frac{z^{2}}{2 h_{\mathrm{i}}^{2}}\right)$,

where the density scale height $h_{\mathrm{i}}$ is linked to the sound speed $c_{\mathrm{i}}$ and the gravitational field $g_{z}$ by

$h_{\mathrm{i}}=c_{\mathrm{i}}\left(\frac{\partial g_{z}}{\partial z}\right)^{-1 / 2}$.

We consider the gravitational temperature $T_{\mathrm{g}}$ at which the thermal energy of a particle balances the gravitational energy, given by

$k_{\mathrm{B}} T_{\mathrm{g}}=\frac{G M_{*} m_{\mathrm{H}}}{r}$, 
where $m_{\mathrm{H}}$ is the atomic mass of hydrogen. Then,

$h_{\mathrm{i}}=r \sqrt{\frac{T_{\mathrm{i}}}{\mu_{\mathrm{i}} T_{\mathrm{g}}}}$,

where $\mu_{\mathrm{i}}$ is the mean molecular mass in the mid-plane of the disc.

In the standard disc model by Lynden-Bell \& Pringle (1974), the mass column $\Sigma$ is linked to the uniform accretion rate $\dot{M}$ by

$\Sigma=\frac{\dot{M}}{3 \pi v_{\mathrm{i}}} f$

where $v_{\mathrm{i}}$ is the vertically averaged viscosity.

In order to go further, we need a prescription for the kinematic viscosity. Shakura \& Sunyaev (1973) use the so-called $\alpha$-prescription:

$v_{\mathrm{i}}=\alpha c_{\mathrm{i}} h_{\mathrm{i}}$,

where $\alpha$ is a dimensionless factor ranging from $10^{-4}$ to $10^{-1}$. If we ignore the thin outer layer, the viscosity is uniform over the entire height because of the isothermal assumption. So, Eq. (13), together with Eq. (14), becomes

$\Sigma=\Sigma_{0}\left(\frac{\mu_{\mathrm{i}} T_{\mathrm{g}}}{T_{\mathrm{i}}}\right), \quad \Sigma_{0}=\frac{\dot{M} f}{3 \Omega_{\mathrm{K}} \alpha \pi r^{2}}$.

The mass column also meets

$\Sigma=2 \int_{0}^{+\infty} \rho(z) \mathrm{d} z$

so that, with Eqs. (13) and (9)

$\rho_{\mathrm{i}}=\rho_{0}\left(\frac{\mu_{\mathrm{i}} T_{\mathrm{g}}}{T_{\mathrm{i}}}\right)^{\frac{3}{2}}, \quad \rho_{0}=\sqrt{\frac{2}{\pi}} \frac{\dot{M} f}{6 \Omega_{\mathrm{K}} \alpha \pi r^{3}}$.

In order to keep an analytical description, we assume that the opacity $\kappa$ locally follows a power law:

$\kappa(\rho, T)=k \rho^{l} T^{m}$.

We now assume that the outer layer has an optical depth much smaller than that of the inner layer, that is $\tau_{\mathrm{e}} \ll \tau_{\mathrm{i}}$. This is indeed true in optically thick regions of the disc, where $\tau_{\mathrm{i}} \gg 1$ and $\tau_{\mathrm{e}} \ll 1$. In optically thin regions, the validity of this approximation must be self-consistently checked after computation. With such an approximation, the optical depth of the disc can be written as if the outer layer were absent:

$\tau_{\mathrm{i}}=\int_{0}^{+\infty} \rho(z) \kappa(z) \mathrm{d} z$

so that

$\tau_{\mathrm{i}}=\sqrt{\frac{\pi}{2(l+1)}} k \rho_{\mathrm{i}}^{l+1} T_{\mathrm{i}}^{m} h_{\mathrm{i}}$.

The corrective term $\Delta \tau_{\mathrm{i}}$ depends on the distribution $\vartheta$ of the viscous heating. Paper I gives

$$
\begin{aligned}
\vartheta(z) & =\frac{\int_{z}^{+\infty} \rho(\zeta) v(\zeta) \mathrm{d} \zeta}{\int_{0}^{+\infty} \rho(\zeta) v(\zeta) \mathrm{d} \zeta}, \\
\Delta \tau_{\mathrm{i}} & =\int_{0}^{+\infty} \rho(z) \kappa(z) \vartheta(z) \mathrm{d} z .
\end{aligned}
$$

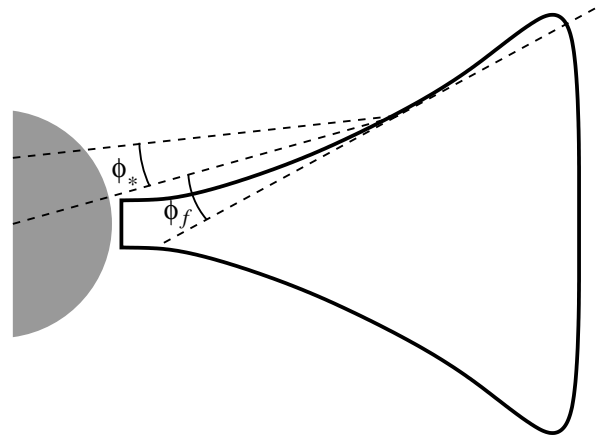

Fig. 2. Incidence angle of stellar radiation onto the disc.

After some calculations we finally obtain

$\tau_{\mathrm{i}}-\Delta \tau_{\mathrm{i}}=\sqrt{\frac{\pi}{2}} \xi_{l} \rho_{\mathrm{i}}^{l+1} T_{\mathrm{i}}^{m} h_{\mathrm{i}}$

with

$\xi_{l}=\frac{1}{\sqrt{l+1}}-\frac{1}{\sqrt{l+2}}$.

We call $\omega$ the ratio of opacity for radiation reprocessed by the outer layer to opacity for stellar radiation. $\omega$ is smaller than unity. With such a definition, the optical depth of the outer layer is

$\tau_{\mathrm{e}} \approx \omega \phi$.

The incidence angle of stellar radiation onto the disc is related to the flaring of the disc. Yet, in a flat disc, this incidence is nonzero because of the vertical extent of the star. Figure 2 shows that $\phi$ is the sum of two contributions: the mean extent of the star $\phi_{*}$ and the tilt of the disc surface toward the star $\phi_{\mathrm{f}}$. Since the star is not a point-like source, we need to integrate over the stellar surface to find $\phi_{*}$. We introduce the flaring angle as the logarithmic variation of the disc thickness $\mathcal{H} / r$ with respect to the radial location:

$\gamma=\frac{\partial \ln \mathcal{H}}{\partial \ln r}-1$.

$\gamma$ represents the variation of the opening angle with the distance to star, i.e. $\mathcal{H} / r \sim r^{\gamma}$. If the opening angle of the disc is constant, e.g. in a flat disc, $\gamma$ is zero. With such a definition, the incidence angle is

$$
\phi=\phi_{\mathrm{f}}+\phi_{*}
$$

with

$\phi_{\mathrm{f}}=\gamma \frac{\mathcal{H}}{r}$,

$\phi_{*} \approx \frac{4}{3 \pi} \frac{r_{*}}{r}$.

The expression of $\phi_{*}$ is an asymptotic value at large $r$, as used in Chiang \& Goldreich (1997). Then, we need to connect $\mathcal{H}$ to the previously determined scale height $h_{\mathrm{i}}$ and use the closure relation

$\mathcal{H}=Q h_{\mathrm{i}}$. 
Chiang \& Goldreich (1997) notice that $Q$ is a slowly varying quantity close to 4 and imposed $Q=4$ over the disc. D'Alessio et al. (1999) consistently determine the location of the outer layer, and we used their results for the fiducial model to derive that $Q$ ranges from 3 to 5 (see Fig. 5h).

Finally,

$\tau_{\mathrm{e}}=\omega\left(\phi_{*}+\gamma Q \sqrt{\frac{T_{\mathrm{g}}}{\mu_{\mathrm{i}} T_{\mathrm{i}}}}\right)$.

\subsection{Implicit equations governing the structure}

First, we use the previous developments to derive the mid-plane temperature $T_{\mathrm{i}}$ as a function of the disc parameters. Second, we derive the flaring index on which the temperature also depends. Last, we determine a boundary condition on $\mathcal{H}$ that allows to derive it over the while disc using $\mathcal{H} / r \propto r^{\gamma}$.

\subsubsection{Mid-plane temperature}

All quantities of Eq. (8) have been expressed in terms of $T_{\mathrm{i}}$ and $\mu_{\mathrm{i}}$, of the physical constants of the disc, and of the quantities $\gamma$ and $Q$. Therefore, we derive an equation on $T_{\mathrm{i}}$

$T_{\mathrm{i}}^{4}=\sum_{k} t_{k}\left(r, T_{\mathrm{i}}(r)\right)^{4}$

with

$t_{1}\left(r, T_{\mathrm{i}}\right)^{4}=\frac{3}{8} \xi_{l} T_{\mathrm{v}}^{4} T_{\mathrm{g}}^{1+\frac{3}{2} l} \Sigma_{0} \rho_{\mathrm{i}}^{l} T_{\mathrm{i}}^{-\frac{3}{2} l+m-1}$,

$t_{2}\left(r, T_{\mathrm{i}}\right)^{4}=\frac{1}{2} T_{\mathrm{eq}}^{4} T_{\mathrm{g}}^{-\frac{1}{2}} \gamma Q \mu_{\mathrm{i}}^{-\frac{1}{2}} T_{\mathrm{i}}^{\frac{1}{2}}$,

$t_{3}\left(r, T_{\mathrm{i}}\right)^{4}=\frac{1}{2} T_{\mathrm{eq}}^{4} \phi_{*}$,

$t_{4}\left(r, T_{\mathrm{i}}\right)^{4}=T_{\mathrm{a}}^{4}$.

Equation (30a) states that the mid-plane temperature is the sum of different heating processes. $t_{k}$ represents the thermal contribution of the $k$ th process. $t_{1}(r)$ is the mid-plane temperature for an optically thick disc dominated by viscous heating; $t_{2}(r)$ is the mid-plane temperature for a flared disc dominated by stellar irradiation, if the geometrical extent of the star is ignored due to the distance; $t_{3}(r)$ is the mid-plane temperature for a flat disc dominated by stellar irradiation; $t_{4}(r)$ is the mid-plane temperature in a disc thermalised with the ambient medium. The radial and thermal dependencies of the contributions $t_{k}$ are

$t_{1}\left(r, T_{\mathrm{i}}\right)^{4} \sim r^{-3 l-9 / 2} f(r)^{l+2} T_{\mathrm{i}}^{-3 / 2 l+m-1}$,

$t_{2}\left(r, T_{\mathrm{i}}\right)^{4} \sim r^{-3 / 2} T_{\mathrm{i}}^{1 / 2}$,

$t_{3}\left(r, T_{\mathrm{i}}\right)^{4} \sim r^{-3}$,

$t_{4}\left(r, T_{\mathrm{i}}\right)^{4} \sim 1$.

\subsubsection{Flaring index}

The case of the flaring is stlightly more complicated. In Chiang $\&$ Goldreich (1997), the disc thickness was both a power law of the distance to the $\operatorname{star}\left(\mathcal{H} \propto r^{9 / 7}\right.$ at large radii) and proportional to the scale height $h_{\mathrm{i}}$. In presence of viscous dissipation, it is no longer possible to ensure that both properties are met, since the scale height is not a simple power law. We investigate three possibilities:

1. The thickness is proportional to the scale height (model 1).

2. The thickness is given by the same law as in the passive disc (model 2).

3. The disc is flat, which occurs if the dust grains settle in the mid-plane (model 3).

The flaring index of model 1 can be expressed as the average of the specific flaring indices $\gamma_{k}$ imposed by the various heating processes weighted by their characteristic temperatures $t_{k}$ (see Appendix B for a demonstration).

$\gamma=\frac{\sum_{k} \gamma_{k} t_{k}^{4}}{\sum_{k} t_{k}^{4}}$

with

$\gamma_{1}=\frac{1-3 l-2 m}{20+6 l-4 m}+\frac{l+2}{20+6 l-4 m} g$,

$\gamma_{2}=\frac{2}{7}$

$\gamma_{3}=\frac{1}{8}$

$\gamma_{4}=\frac{1}{2}$

where

$g=\frac{1-f}{f}$

arises from the departure of viscous heating from the radial power law in $r^{-3}$. This effect occurs close to the star and is characterised by $f$ (Eqs. (1) and (2)). $\gamma_{1}$ is the flaring index for an optically thick active disc, therefore depending on the opacity law; $\gamma_{2}$ the flaring index in a passive disc, if the geometrical extent of the star is ignored; $\gamma_{3}$ the flaring index in a flat passive disc; $\gamma_{4}$ the flaring index in a disc thermalised with the ambient medium.

In Chiang \& Goldreich (1997), the thickness meets $\mathcal{H} \propto r^{\gamma}$, with $\gamma=1 / 8$ close to the star and $\gamma=2 / 7$ at large radii, depending whether the dominant effect in stellar light reprocessing is the extent of the star $\left(t_{3}\right)$ or the disc flaring $\left(t_{2}\right)$. We have assumed in model 2 that, even in the presence of viscosity, we can still write

$\gamma=\frac{\gamma_{2} t_{2}^{4}+\gamma_{3} t_{3}^{4}}{t_{2}^{4}+t_{3}^{4}}$

which means the disc thickness $\mathcal{H}$ is only governed by the irradiation terms $\left(t_{2}\right.$ and $\left.t_{3}\right)$ and varies from $1 / 8$ to $2 / 7$ from the central parts to the outer ones.

In model 3, the disc is flat, that is $\gamma=0$. 


\subsubsection{Disc thickness on the outer edge}

In model 1 we have assumed, like in Chiang \& Goldreich (1997), that the closure relation $\mathcal{H}=Q h_{\text {i }}$ applies, with $Q$ constant over the disc. The authors took $Q=4$, which is seemingly a good approximation for low-mass $\operatorname{discs}\left(\dot{M} \approx 10^{-8} M_{\odot} / \mathrm{yr}\right)$ but which might no longer be correct over the wide range of masses that we shall consider $\left(\dot{M} \approx 10^{-8}-10^{-4} M_{\odot} /\right.$ yr $)$. For this reason we prefer to determine the value of $Q$, and chose to carry out the calculation at the outer edge of the disc.

In model 2, the thickness is no longer proportional to the scale height, so that we have to determine $\mathcal{H}$ with the relation $\frac{\mathrm{d} \ln \mathcal{H}}{\mathrm{d} \ln r}=\gamma+1$, that requires a boundary condition, and therefore determine $\mathcal{H}$ at the other edge of the disc. We perform this calculation by computing $Q=\mathcal{H} / h_{\mathrm{i}}$.

So, we need to determine the value of $Q$ at the outer boundary of the disc in both models, which leads us to approximately solving the vertical hydrostatic equilibrium and the radiative transfer of the incoming radiation. We obtain

$Q=\sqrt{\frac{2}{\delta_{\rho}(Q)} \ln \left(\frac{\kappa_{\mathrm{e}} \rho_{\mathrm{i}} r}{\gamma Q \omega} \sqrt{\frac{T_{\mathrm{e}}}{T_{\mathrm{i}}} \delta_{h}(Q)}\right)}$,

where $\delta_{h}$ and $\delta_{\rho}$ are corrections due to the deviation of the gravity field from the linear law $g_{z} \propto z$ when the elevation becomes of the order of $r$ :

$$
\begin{aligned}
& \delta_{\rho}(Q)=2\left(\frac{r}{Q h_{\mathrm{i}}}\right)^{2}\left[1-\left(1+\left(Q h_{\mathrm{i}} / r\right)^{2}\right)^{-1 / 2}\right], \\
& \delta_{h}(Q)=\left(\left(1+\left(Q h_{\mathrm{i}} / r^{2}\right)\right)^{3 / 2} .\right.
\end{aligned}
$$

A demonstration can be found in Appendix C.

\section{Numerical approach}

Though analytical, the expressions of $T_{\mathrm{i}}$ and $\gamma$ do not allow direct determination, because the equations giving $\gamma$ and $T_{\mathrm{i}}$ are coupled and the opacities do not follow a unique power law over the whole disc. This section first describes the numerical method we used for the derivation of the structure. Then, we briefly explain who the observables (SEDs and visibilities) have been derived.

\subsection{Opacities}

Because the heating and the flaring of the disc is dependent on opacities, their adequate description is mandatory. The computation of the SED and image requires monochromatic opacities that we took from two sources:

- At low temperatures $(T \lesssim 1800 \mathrm{~K})$ the opacity is dominated by the dust. We use opacities computed by Henning \& Stognienko (1996) for a mixture of inhomogeneous aggregates; they include continuum emission and the silicate feature in the mid-infrared.

- At higher temperatures $(T \gtrsim 1800 \mathrm{~K})$ the opacity comes from to the gas. We could not find such opacities; instead, we used piecewise power laws $\kappa_{\lambda}(\rho, T)=\kappa_{0}(\rho, T) \lambda^{-p}$, and chose $(\rho, T)$ and $q$, so that they approximately match
Rosseland opacities by Bell \& Lin (1994) and Planck opacities by Henning \& Stognienko (1996). Despite of this poor description, the influence on the observables is small because it only concerns the inner disc, where both optical depth $(\tau \gg 1)$ and thickness $(\mathcal{H} \ll r)$ are not critical.

These opacities were smoothed in order to avoid numerical instabilities between the different domains; it is justified since we use a vertically averaged structure in both layers.

The structure requires the Planck and Rosseland opacities, as well as the determination of the opacity ratio $\omega$. They have been determined from the monochromatic opacities as explained in Appendix A. In order to keep the power law opacities formalism, we determined the local equivalent $\kappa_{\mathrm{R}} \propto \rho^{l} T^{m}$ with

$$
\begin{gathered}
l=\frac{\partial \log \kappa_{\mathrm{R}}}{\partial \log \rho}, \\
m=\frac{\partial \log \kappa_{\mathrm{R}}}{\partial \log T} .
\end{gathered}
$$

\subsection{Physical conditions in the disc}

The disc is divided in concentric cylinders and the two-layer formalism is applied for each one: it consists in computing $t_{k}$, refining the values for $T_{\mathrm{i}}$ and $\gamma$ (Eqs. (30) and (32)), computing the corresponding opacities with the associated $l$ and $m$ (Eq. (38)), deriving $\mathcal{H}$, and iterating until convergence. In model 1 , the computation is carried out in ascending order of radius Cylinders shadowed by inner ones do not receive stellar light and are determined with the same equations, but using $T_{\star}=0 \mathrm{~K}$. $\mathcal{H}$ is determined as $\mathcal{H}=Q h$ with $Q$ constant; the self-consistent determination of $Q$ at the outer edge has not been implemented so far. In model 2, $Q$ is determined at the outer edge (Eq. (28)), then the computation is performed from outer to inner cylinders. For each cylinder, $\mathcal{H}$ is given by the thickness of the enclosing cylinder $\mathcal{H}^{\text {out }}$ using

$\mathcal{H}=\mathcal{H}^{\text {out }}\left(\frac{r}{r^{\text {out }}}\right)^{1+\gamma}$.

The validity of the derived structure was checked with two tests. On one hand, when setting $\gamma=0$ and $\tau_{\mathrm{e}}=1$, the results are consistent with those of the standard disc as determined in Bertout et al. (1988). On the other hand, the model locally determines the flaring index (Eq. (32)) without using the radial structure, and we checked that the derived radial structure fulfilled $\frac{\mathrm{d} \mathcal{H}}{\mathrm{d} r}=\gamma$.

\subsection{Spectral energy distributions}

In order to compute a synthetic image or a SED, we divide each disc annulus in angular sectors. For each sector we determine the emergent flux in the observer's direction as the sum of three contributions: the contributions of the outer layers $F_{\lambda}^{\mathrm{e}}$, of the inner layer $F_{\lambda}^{\mathrm{i}}$, and of simple isotropic scattering of stellar radiation by the surface of the disc $F_{\lambda}^{\mathrm{s}}$. These fluxes depend on the wavelength-dependent optical thicknesses $\tau_{\lambda}^{\mathrm{i}}$ and $\tau_{\lambda}^{\mathrm{e}}$ of the inner and outer layers. The incidence of the line of sight onto 
the visible surface of the disc is given by its angle $i$, and its incidence onto the opposite surface by $i^{\prime}$.

$F_{\lambda}=F_{\lambda}^{\mathrm{i}}+F_{\lambda}^{\mathrm{e}}+F_{\lambda}^{\mathrm{s}}$

with

$F_{\lambda}^{\mathrm{i}}=\left(1-\mathrm{e}^{-\frac{2 \tau_{\lambda}^{\mathrm{i}}}{\cos i}}\right) \mathrm{e}^{-\frac{\tau_{\lambda}^{\mathrm{e}}}{\cos i}} B_{\lambda}\left(T_{\lambda}^{\mathrm{phot}}(i)\right)$,

$F_{\lambda}^{\mathrm{e}}=\left(\left(1-\mathrm{e}^{-\frac{\tau_{\lambda}^{\mathrm{e}}}{\cos i}}\right)+\left(1-\mathrm{e}^{-\frac{\tau_{\lambda}^{\mathrm{e}}}{\cos i^{\prime}}}\right) \mathrm{e}^{-\frac{2 \tau_{\lambda}^{\mathrm{i}}}{\cos i}}\right) B_{\lambda}\left(T_{\mathrm{e}}\right)$,

$F_{\lambda}^{\mathrm{s}}=\frac{\mathcal{A} \sin (\phi)}{2}\left(\frac{r_{*}}{r}\right)^{2} B_{\lambda}\left(T_{*}\right)$,

where $B_{\lambda}$ is the black-body function. $T_{\lambda}^{\text {phot }}(i)$ is the temperature of the inner layer at optical depth $\cos i$ at wavelength $\lambda$ if $\tau_{\lambda}^{\mathrm{i}}>$ $\cos i$, and its mid-plane temperature in the other case.

This determination allows a fast computation but is only an approximation. It is only valid when the outer parts of the disc shadows neither the star nor other parts of the disc; therefore a more adequate radiative transfer determination must be carried out for edge-on discs, which is beyond the scope of this paper.

As a test, we have checked that the bolometric flux predicted by the SED is consistent with the one predicted by the effective temperatures of the heating processes. We noticed discrepancies of $2-3 \%$ in most cases, up to $8 \%$ in a few ones; they are connected to the use of the mean opacities $\kappa_{\mathrm{R}}$ and $\kappa_{\mathrm{P}}$ in the structure calculation.

\subsection{Visibilities}

The image and visibilities are determined using the same method as for the SED. However, optical visibilities at long baselines can be both sensitive to large-scale structures up to $1^{\prime \prime}$ (e.g. Palomar Testbed Interferometer field of view) and to small scale structures down to 0.1 mas. As the visibility function is the Fourier transform of the image, a straightforward determination would require a huge number of image pixels $\left(\approx 10^{4} \times 10^{4}\right)$.

As one of the goals of the present model is rapidity, we avoid this determination. Our image $I(x, y)$ contains the central structure with about $10^{3} \times 10^{3}$ pixels. All flux falling outside this image is integrated as a single value $F_{\text {out }}$. Then, the nonnormalised visibility $\mathcal{V}$ is given by

$\mathcal{V}(u, v)= \begin{cases}\tilde{I}(0,0)+F_{\text {out }} & \text { if } u=v=0 \\ \tilde{I}(u / \lambda, v / \lambda) & \text { otherwise }\end{cases}$

where $\tilde{I}$ is the Fourier transform of $I$. This equation states that the $F_{\text {out }}$ contribution cancels as soon as the baseline is nonzero, which means that the outer parts of the disc are fully resolved for any arbitrarily small baseline and would produce a visibility of 0 if they were alone. Two approximations are made: (i) the outer parts of the disc do not contain small-scale structures (ii) for too small baselines Eq. (41) is not valid. Our model does include neither irregularities nor steep variations in the outer parts of the disc so that approximation (i) is valid. The smallest baseline for which Eq. (41) is valid is determined
Table 1. Parameters used for the fiducial model.

\begin{tabular}{ll}
\hline \hline Parameters & fiducial model \\
\hline$r_{*}\left(R_{\odot}\right)$ & 2.0 \\
$r_{\min }\left(R_{\odot}\right)$ & 6.0 \\
$r_{\max }(\mathrm{AU})$ & 100 \\
$M_{*}\left(M_{\odot}\right)$ & 1.0 \\
$\dot{M}\left(M_{\odot} / \mathrm{yr}\right)$ & $1.0 \times 10^{-8}$ \\
$T_{*}(\mathrm{~K})$ & 4000 \\
$i\left(^{\circ}\right)$ & 0 \\
$A_{V}(\mathrm{mag})$ & 0.0 \\
$\mathcal{A}$ & 0.2 \\
$\alpha$ & $1 \times 10^{-2}$ \\
\hline
\end{tabular}

by the field of view of the image ${ }^{1}$ and we choose this field in order to produce correct results for the smallest baselines used in long-base optical interferometry $(\approx 10 \mathrm{~m})$.

\section{Model analysis}

In this section, we study the influence of disc flaring hypotheses, compare our model with other authors' models, analyse the influence of different heating processes, show the influence of viscosity prescription and examine the influence of some disc parameters on both the structure and the observables. For the sake of comparison, authors compute a fiducial model, typical of a T Tauri star; its parameters are displayed in Table 1.

From now on, we use model 2 in our study, unless specified otherwise (Sect. 4.1 and appendices).

\subsection{Importance of disc flaring hypotheses}

Figure 3 compares the structure of the fiducial $\mathrm{T}$ Tauri disc in three cases: the disc thickness is proportional to the scale height (model 1), the disc thickness is given by the reprocessing terms only (model 2), and the disc is flat (model 3 ).

The major difference between models 1 and 2 is the presence of self-shadowing in the first one: at a radius of a few AUs, the surface temperature of model 1 drops because the disc is not directly illuminated by the star (see middle panel of Fig. 3 ). The cause is a decrease of the relative scale height $h / r$ (see right panel on the same figure), hence a similar behaviour of the disc surface because of the proportionality $\mathcal{H} \propto h$. A contrario, model 2 assumes a thickness increasing faster than $r$ $\left(\mathcal{H} \propto r^{1+\gamma}\right)$, so the surface is always illuminated.

Model 3 is a flat disc, therefore it catches much less light from the central star: the outer parts are much cooler (Fig. 3, $T_{\mathrm{i}}$, left panel). If the surface has the same temperature (cf. $T_{\mathrm{e}}$, middle panel), it produces a much smaller flux because the outer layer happens to be extremely thin. Such a disc has a lesser scale height because it is cooler (cf. $h / r$, right panel).

As we shall see in Sect. 4.3, numerical simulations tend to prove that there is no self-shadowing effect in $\mathrm{T}$ Tauri discs. D'Alessio et al. (1999) establishes that even with a scale

\footnotetext{
1 The visibility is the discretised Fourier transform of the image and the smallest non-zero baselines obtained are given by $B=\lambda / \vartheta$, where $\vartheta$ is the field of the image. As a consequence Eq. (41) is only valid for baselines $\gtrsim B$.
} 


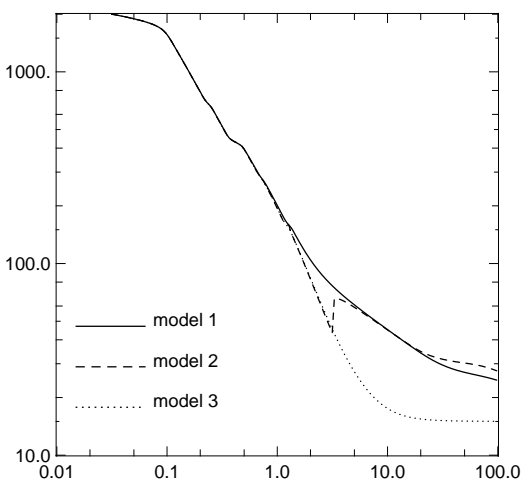

a $T_{\mathrm{i}}$

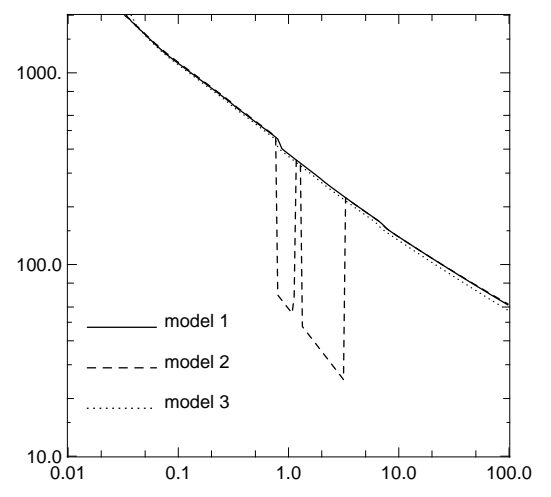

b $T_{\mathrm{e}}$

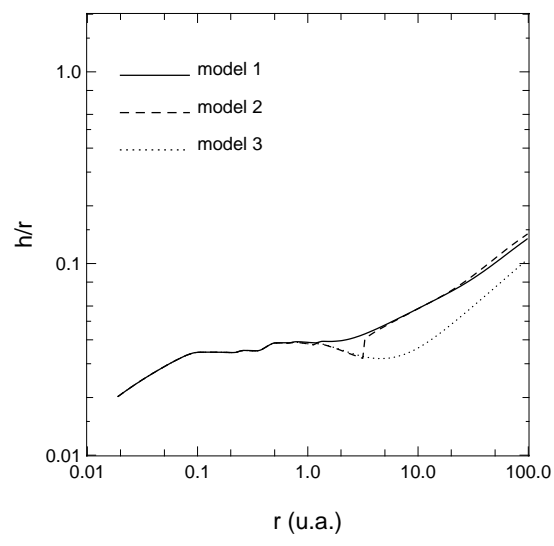

c $h / r$

Fig. 3. Influence of disc flaring hypotheses on the structure. Model 1: thickness proportional to the scale height, model 2: self-similar thickness, model 3: flat disc. Left: mid-plane temperature, middle: surface temperature, right: relative scale height.

height flaring inwards, there is still enough material at large $z$ to catch stellar light, so that all parts of the disc are illuminated. Moreover, we noticed that model 2, with the ad hoc flaring index, compares much better with other simulations than model 1. So, we shall continue our study with model 2.

\subsection{Viscosity prescription}

We compare the standard $\alpha$ viscosity prescription with the $\beta$ prescription derived from laboratory experiments by Huré et al. (2001). $\beta$ is defined as

$v_{\mathrm{i}}=\beta \Omega_{\mathrm{K}} r^{2}$

and is a slowly varying factor ranging from $10^{-6}$ to $10^{-2}$. We keep it constant over the disc.

We chose $\beta=10^{-5}$ so that the viscosity $v_{\mathrm{i}}$ has the same order of magnitude as with the $\alpha$-model in the central parts of the disc. This prescription gives much higher column density and disc mass in the outer parts of the disc, as displayed in Fig. 4. Note that the impact of viscosity on the observables is small, since both viscous effective temperature and reprocessing flux do not directly depend on it. In the mid-IR, the SED is only slightly affected, via a subtle modification of the disc thickness $\mathcal{H}$, with an impact of the reprocessing temperature. Larger wavelength observations $(>100 \mu \mathrm{m})$ probe the outer parts of the disc, and should become more sensitive to the influence of the viscosity law, because the flux emerging from these optically thin parts is proportional to $\kappa \Sigma T_{\mathrm{i}}$.

\subsection{Comparison with other models}

We computed the two-layer structure of the fiducial model (Table 1) and compare it model with others listed in Table 2 together with their main characteristics. Figure 5 displays some physical conditions describing the radial structure of the fiducial disc, as forecast by these models. As a general result, despite of the approximation made, our model is in good agreement with previous ones.
Table 2. Disc model characteristics compared in Sect. 4.3. We mention whether stellar light reprocessing, viscous heating and convective transport are taken into account, whether the vertical transfer is solved or vertically averaged, and the viscosity prescription used.

\begin{tabular}{llllll}
\hline \hline & $\mathrm{a}$ & $\mathrm{b}$ & $\mathrm{c}$ & $\mathrm{d}$ & $\mathrm{e}$ \\
\hline reprocessing & yes & no & yes & yes & no \\
viscosity & yes & yes & yes & no & yes \\
convection & no & no & no & yes & no \\
vertical transfer & no & 1D & 1D & no & $1 \mathrm{D}$ \\
viscosity prescription & $\alpha, \beta$ & $\alpha, \beta$ & $\alpha$ & - & $\alpha$ \\
\hline a: Present model & & & & & \\
b: Paper II & & & & & \\
c: D'Alessio et al. (1999) & & & & \\
d: Chiang \& Goldreich (1997) & & & & \\
e: Bell et al. (1997) & & & & & \\
\hline
\end{tabular}

Some discrepancies with the Bell et al. (1997) and Chiang \& Goldreich (1997) models come from different heating hypotheses. Chiang \& Goldreich (1997) obtain lower values for temperatures in the inner parts of the disc, and higher mass columns since $\Sigma v$ is constant (see Figs. 5a and $5 \mathrm{~g}$ ). The reason is that they do not include viscous heating, predominant in the first AUs of T Tauri discs (see Fig. 6 for $r \lesssim 1 \mathrm{AU}$ ). In the outer parts of the disc, dominated by reprocessing, the Bell et al. (1997) model differs significantly because it does not take this process into account.

Our model and that of D'Alessio et al. (1999) present very close predictions in terms of central temperature, surface temperature or scale height. Huré \& Galliano (2001) already noticed that the flat vertically averaged disc model is a good approximate of an active disc; we have now demonstrated that a two-layer model is a faithful description of discs both illuminated by the central star and heated by viscosity. However there are still some discrepancies:

- Our prediction of the disc thickness $\mathcal{H}$ is an underestimation in the range $0.1-1 \mathrm{AU}$, so is the effective temperature of reprocessing $T_{\mathrm{r}}$. 

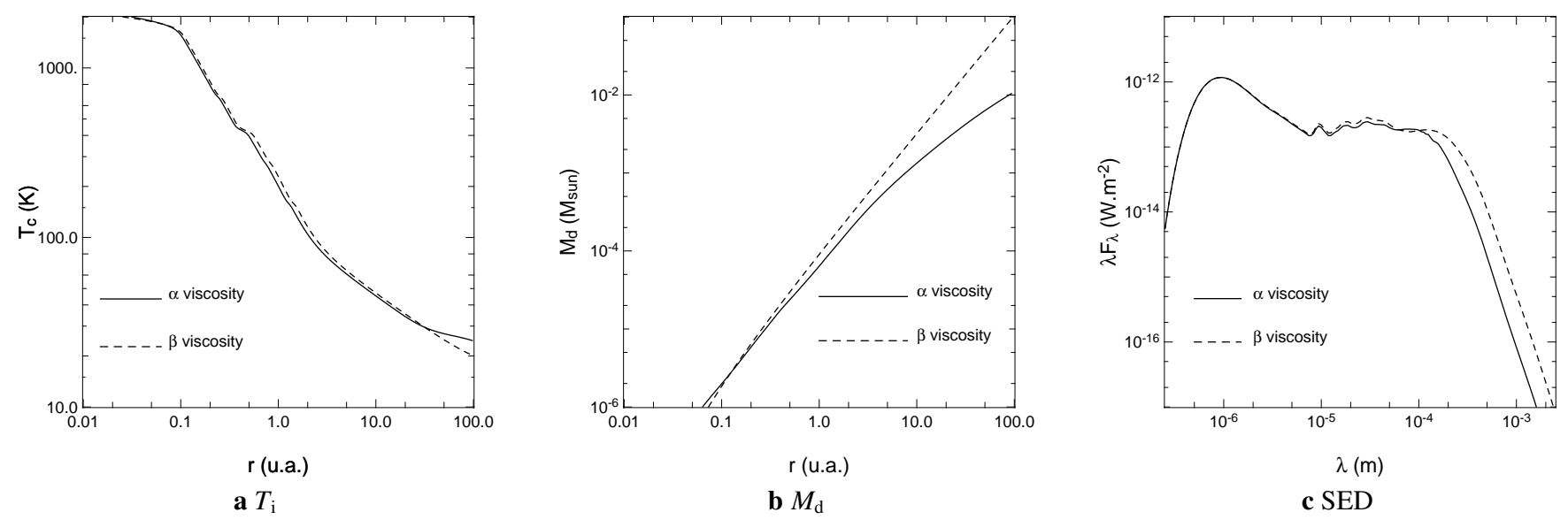

Fig. 4. Influence of the viscosity prescription. Solid lines: $\alpha$-prescription by Shakura \& Sunyaev (1973); dashed lines: $\beta$-prescription by Huré et al. (2001). Right panel: mid-plane temperature vs. radius; middle panel: mid-plane temperature vs. radius; right panel: SED.

- Despite of a smaller effective temperature than in D'Alessio et al. (1999), our SED is a slight overestimation in the range $20-100 \mu \mathrm{m}$. There is a likely reason: the two-layer structure tends to over-estimate the temperature of emission by the outer layer of the disc, hence the far-infrared excess.

- We find important discrepancies in the region $r \lesssim 0.1 \mathrm{AU}$, because our opacities at intermediate temperatures $(T \gtrsim$ $1800 \mathrm{~K}$ ) are different from those of D'Alessio et al. (1999).

These discrepancies do not affect much the results concerning SED and long-baseline visibility model fitting.

\subsection{Location of heating processes}

Figure 6 displays the radial profile of contributions of each heating process to the temperature of a typical $\mathrm{T}$ Tauri disc. The mid-plane temperature of the fiducial model is dominated by reprocessing for $r \gtrsim 2 \mathrm{AU}$ and by viscous dissipation for $r \lesssim 2 \mathrm{AU}$. However, in terms of effective temperature, the disc is dominated by reprocessing at any radial location, with a marginal contribution of viscosity for $r \lesssim 0.2 \mathrm{AU}$. The outer layer is completely dominated by stellar light reprocessing at any distance from the star. One important conclusion to draw is that the notion of a disc dominated by a heating process depends on what quantity we are interested in.

\subsection{Thermal flux vs. scattered light}

Simple disc models often ignore scattering when they focus on the inner parts, but at large radius scattered light is dominant. A reason for this is that the thermal flux decreases exponentially with $r$, since it is proportional to $B_{\lambda}(T(r))$, while reprocessed flux is roughly in proportionnal to $r^{-2}-$ more exactly $\propto r^{-2} \phi$. Figure 7 represents the contribution of thermal and scattered light to the emergent flux from a typical $\mathrm{T}$ Tauri disc, in different spectral bands.

Let us first notice that the flux emerging from a standard "one-layer" disc is dominated by scattered light for $r>$ $0.15 \mathrm{AU}$ in $J, r>0.5 \mathrm{AU}$ in $K$, and $r>20 \mathrm{AU}$ in $N$ (contribution of the inner layer, Fig. 7). Ignoring scattering in AU-scale predictions with the standard model proves out to be a bad approach in the near-IR.

In a two-layer disc, the outer layer is hotter and produces a larger thermal flux (contribution of the outer layer, same figure). Scattering is therefore dominant only at large radii: $r>2 \mathrm{AU}$ in $J, r>8 \mathrm{AU}$ in $K$, and $r \gtrsim 500 \mathrm{AU}$ in $N$, so that ignoring it as a first approach can be an interesting simplification, when dealing with the AU-scale observations provided by IR interferometry, provided that the field of view of the interferometer is limited (see Sect. 4.6.1). In other words, the thermal emission is more extended in a two-layer disc, which implies, for instance, lower optical visibilities.

\subsection{Influence of disc parameters}

Figure 8 displays the influence of four parameters on the disc structure and the observables - the SED and the normalised visibility.

The visibility curves are built using a point-like source, the star, with a flux $F_{*}$, over-imposed on an extended source $F_{\text {disc }}$. At large baselines, the visibility amplitude reaches a plateau yielding information on the flux ratio $|V|^{2} \approx 1 /\left(1+F_{*} / F_{\text {disc }}\right)^{2}$. The higher the plateau, the smaller the ratio $F_{\text {disc }} / F_{*}$. The baseline $B$ at which the plateau is reached gives the disc extent $\vartheta \approx \lambda / B$. The higher the baseline $B$, the smaller the disc.

At first sight the SED and visibilities present general features: the presence of a silicate feature in emission, due to the super-heated outer layer, and a steep drop of visibility when the wavelengths approaches the $N$ band, also linked to the silicates: at $10 \mu \mathrm{m}$ the outer layer becomes suddenly optically thicker and contributes more to the total flux; since it is hotter and therefore presents a larger extent than the inner layer, the disc appears much larger.

\subsubsection{Albedo}

When the albedo varies, the outer parts of the structure and the SED are altered, because reprocessing is lowered. The effect on the structure of the inner parts is not visible, because 


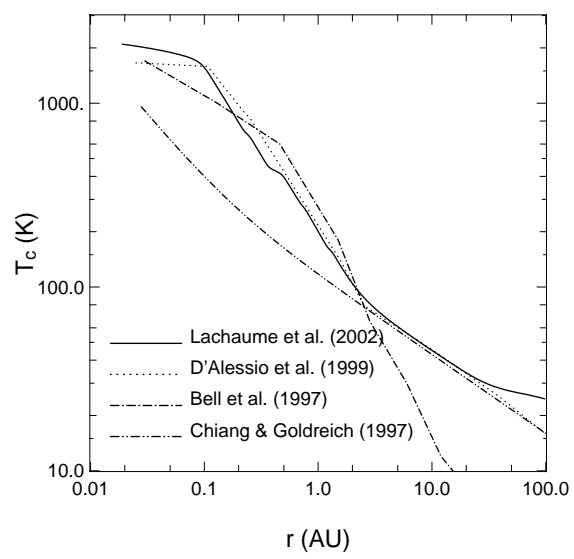

a $T_{\mathrm{i}}$

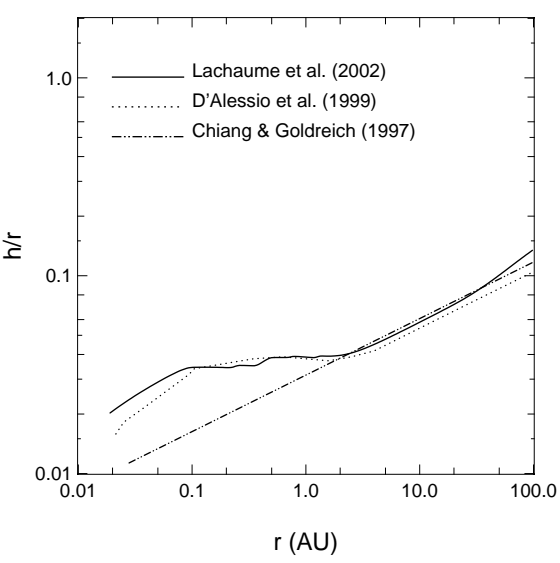

d $h_{\mathrm{i}} / r$

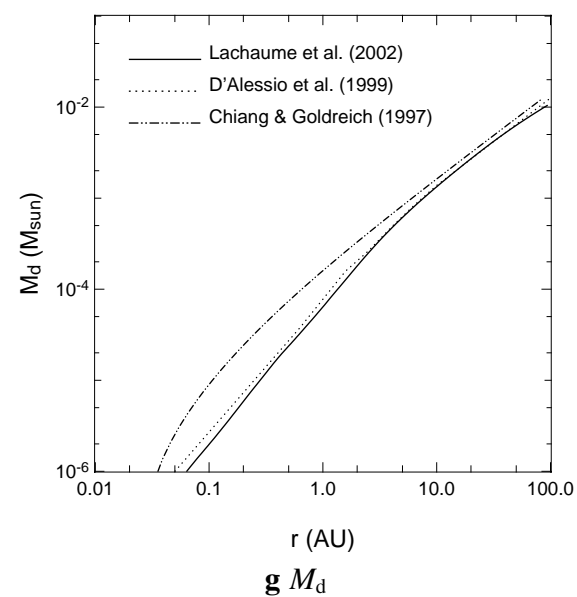

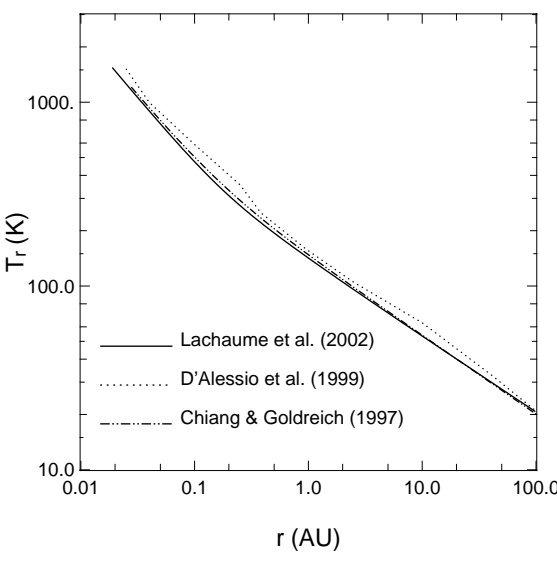

b $T_{\mathrm{r}}$

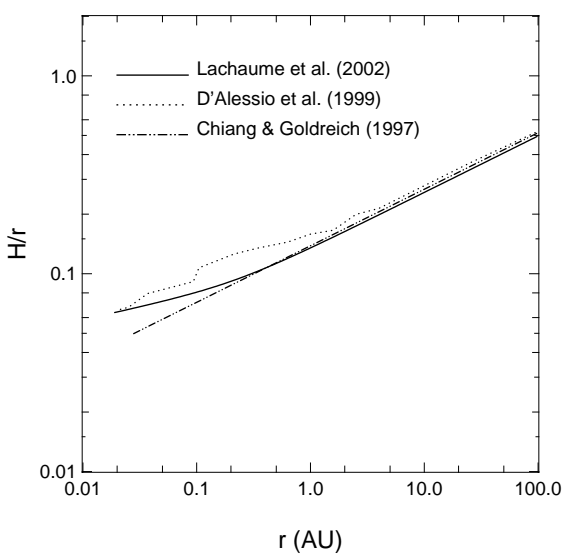

e $\mathcal{H} / r$

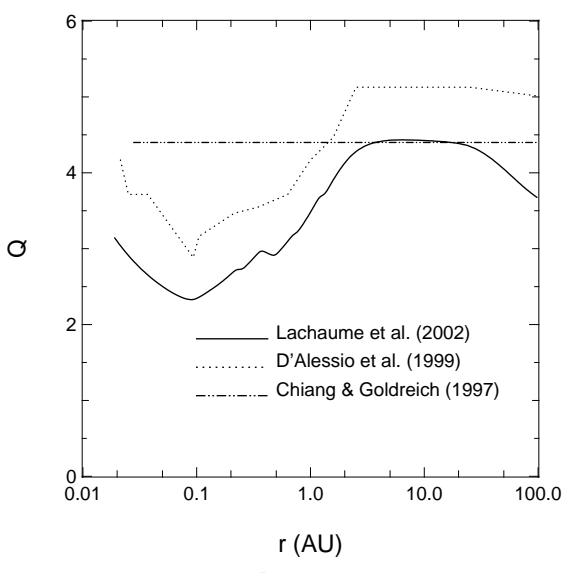

h $Q$

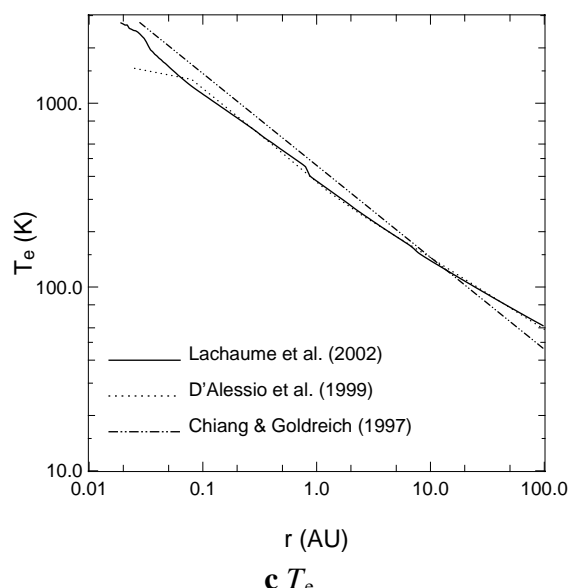

c $T_{\mathrm{e}}$

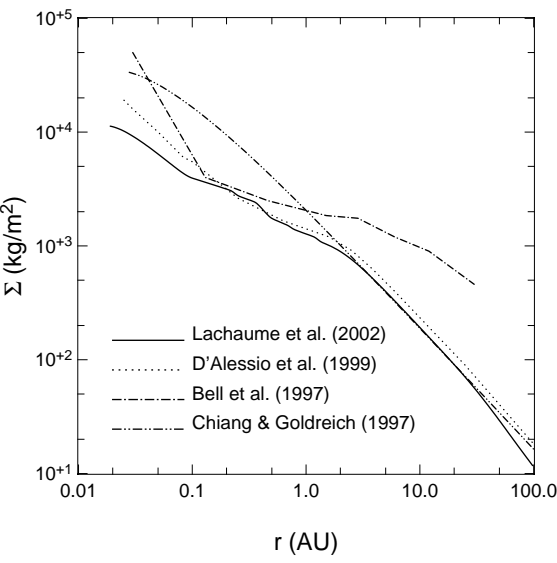

f $\Sigma$

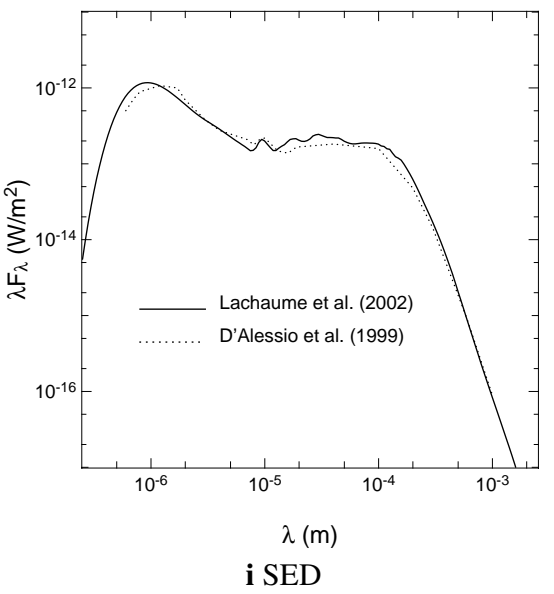

Fig. 5. Comparison between disc models: present work with $\alpha$ and $\beta$ prescription, D'Alessio et al. (1999), Bell et al. (1997) and Chiang \& Goldreich (1997). The panels are: a) mid-plane temperature, b) effective temperature associated to stellar irradiation, c) surface temperature, d) scale height, e) mean height of reprocessing, f) column density, g) cumulative disc mass, h) relative geometrical thickness of the disc, i) spectral energy distribution of the pole-on disc.

reprocessing is not important there. The visible SED is only slightly changed because stellar light dominates scattered light by a factor $>10$.

While one usually ignores scattering in simple disc models, its incidence on the visibility curve can be important. The scattered flux represents about $\mathcal{A} \mathcal{H} / r$ of the stellar flux for a disc with a relative outer geometrical thickness $\mathcal{H} / r$, i.e. $\approx 5$ to $10 \%$ in most discs. At near-infrared wavelengths and for moderate accretion rates, direct stellar flux and disc reprocessed flux are comparable, so scattered light cannot be ignored. Since reprocessing occurs at a large scale (like scattering, see Fig. 6), an extended structure is resolved at very small baselines, hence 


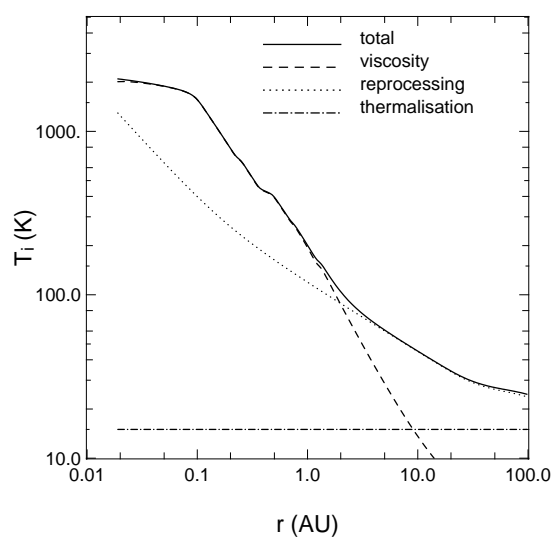

a $T_{\mathrm{i}}$

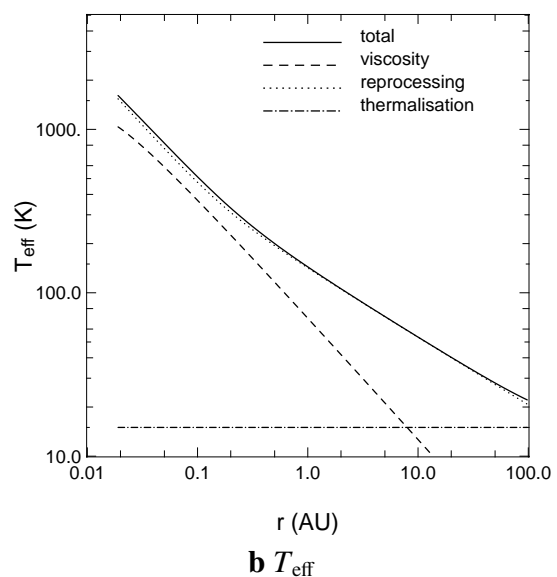

b $T_{\text {eff }}$

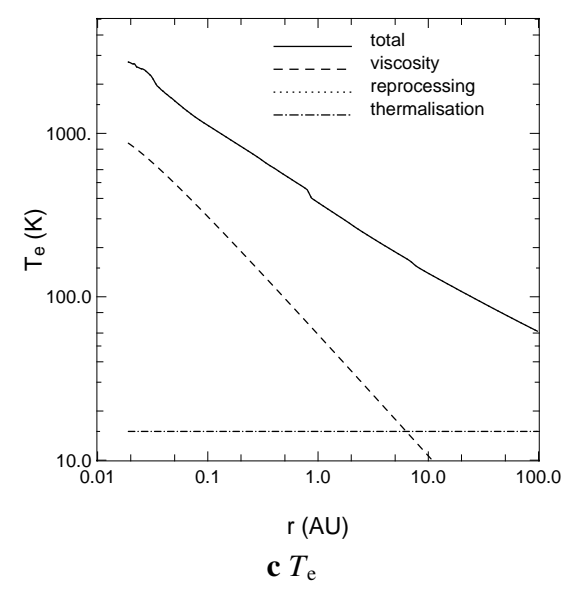

c $T_{\mathrm{e}}$

Fig. 6. Contribution of heating processes: radial profile of these contributions to the temperature of the disc (model 2). Left: mid-plane temperature, middle: surface temperature, right: surface temperature.

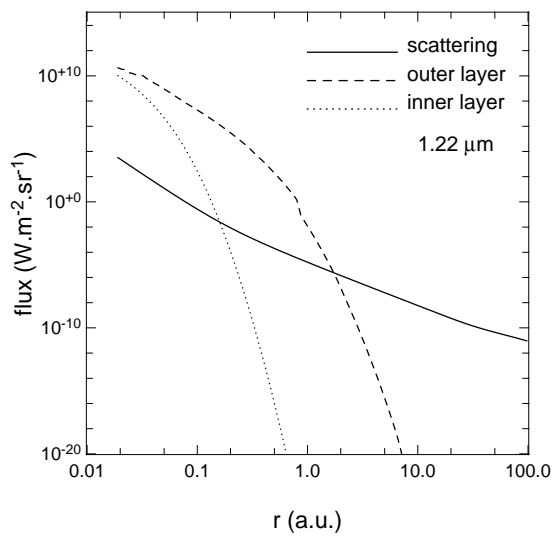

a J band

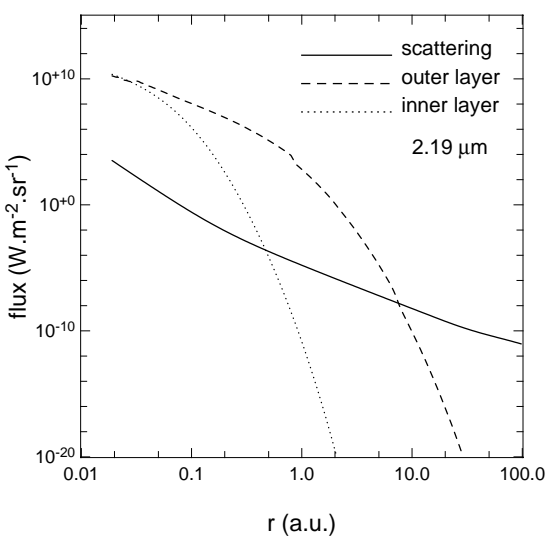

b $\mathrm{K}$ band

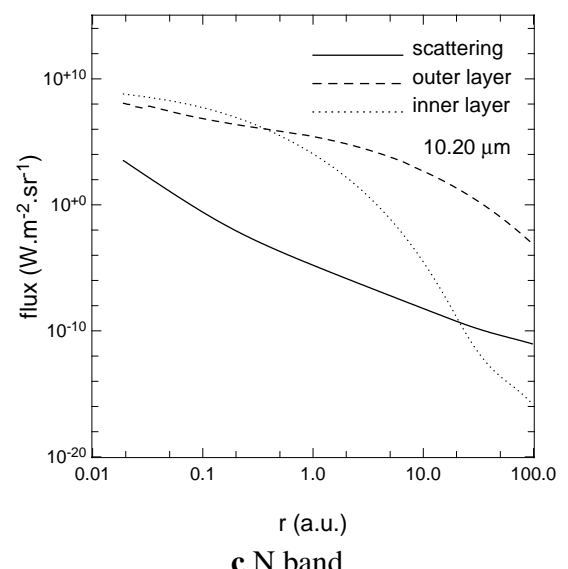

c $\mathrm{N}$ band

Fig. 7. Scattered light and thermal flux from a typical T Tauri disc (model 2) vs. the distance to the star. Left: $J$-band; middle: $K$-band; right: $\mathrm{N}$-band. Solid lines: scattered light; dashed lines: thermal emission of the outer layer; dotted lines: thermal emission of the inner layer.

the visibility drop near the origin. This effect might not be seen if the interferometer field of view is not large enough, hence a possible discrepancy up to 0.1 in normalised visibility.

An important scattering also alters the variation of the visibility with wavelength by a differential effect: scattering is much more important at short wavelengths, where it induces a larger drop in visibility.

\subsubsection{Inner disc radius}

As the inner truncation of the disc increases, the near-infrared part of the SED is depleted, because this part of the spectrum is mostly produced by the hot inner parts of the disc.

The very long baseline $(B>400 \mathrm{~m})$ visibility amplitudes are higher, because the flux of the disc is lower compared to the flux of the star. The visibility plateau is reached for smaller baselines $(\approx 50-100 \mathrm{~m})$ because the disc has a larger mean square dimension. For small baselines, the visibility is lower because the resolved outer parts of the disc have a greater contribution compared to its still unresolved parts.
The inner truncation also affects the variation of visibility with wavelength, because of a resolution effect. At small wavelengths, the resolution is high and visibilities follow the very long baseline trend and the visibility is higher for a large inner radius. At large wavelengths, the resolution is low and the visibility is lower for a large inner truncation.

\subsubsection{Viscosity}

As $\alpha$ increases, the viscosity becomes more efficient. The amount of material needed to produce a given accretion rate decreases. Therefore the optical thickness of the disc becomes lower and the mid-plane temperature follows the same trend. The discrepancy in the IR SED and visibility amplitudes are small, because the effective temperature of viscosity does not directly depend on $\alpha$. However, when $\alpha$ increases, $\Sigma$ decreases, so that the thickness $\mathcal{H}$ also decreases and less stellar light is caught by the disc. Therefore, the mid IR SED is depleted and the visibility is slightly higher. The SED is much more affected in the submillimetre, because it probes the optically thin outer parts of the disc, so that the flux is proportional to $\Sigma \propto \alpha^{-1}$. 
Table 3. Interferometric observations of FU Ori and T Tau stars.

\begin{tabular}{llll}
\hline \hline star & band & instrument & reference \\
\hline FU Ori & $H \& K$ & PTI/IOTA & $a$ \\
T Tau N & $K$ & PTI & $b$ \\
SU Aur & $K$ & PTI & $b$ \\
\hline${ }^{a}$ Malbet et al. (1998); Malbet \& Berger (2002). \\
${ }^{b}$ Akeson et al. (2000, 2002). \\
\hline
\end{tabular}

\subsubsection{Accretion rate}

As the accretion rate increases, the mid-plane temperature is higher in the regions dominated by viscous heating $(r<1-$ $10 \mathrm{AU}$ ), but remains unchanged in the outer regions dominated by reprocessing. The near- and mid-infrared parts of the SED drastically change with accretion rate, since the viscous effective temperature is proportional to the accretion rate. At small accretion rates, the temperature inversion at the surface produces a silicate feature in emission at $10 \mu \mathrm{m}$; when $\dot{M}$ increases, the temperature inversion disappears, because the reprocessing becomes secondary, and the feature disappears. The far-infrared SED does not change much for moderate accretion rates $\left(10^{-9}-10^{-7} M_{\odot} / \mathrm{yr}\right)$, because the regions that significantly contribute at large wavelength are dominated by stellar light reprocessing (almost) independent of the accretion. The slight decrease in the far infrared, when accretion increases, is due to the change of $\mathcal{H}$ with the amount of material.

The visibility presents a lower plateau for larger accretion rates, because the flux from the disc becomes higher compared to the flux from the star. For large accretion rates ( $>10^{-7} M_{\odot} / y r$ ), the plateau is reached for smaller baselines because the mean angular size of the disc is larger (the hotter the disc, the larger the region of emission at a given wavelength). However at small baselines, discs with moderate accretion present a faster visibility drop because the resolved scattered flux is larger compared to the still unresolved inner parts.

\section{Comparison with observations}

We limit our study to TTS and FU Orionis stars for which both SEDs and optical visibilities are available. The sample consists of three objects: FU Ori, SU Aur and T Tau North. Table 3 lists interferometric observations available for these stars. SED measurements have been taken from Gezari et al. (1999). Table 4 lists the physical parameters of the best fits.

Model-fitting of disc visibilities has already been carried out by Malbet \& Berger (2002); Malbet (2002): they consistently fitted the spectrum and $K$ visibilities for FU Ori, with a self-similar flat disc model presenting an effective temperature $\propto r^{-0.75}$ but were not able to reproduce both $H \& K$ visibilities unless assuming a radial temperature law in $r^{-0.4}$ to $r^{-0.6}$, that most physical accretion disc models cannot reproduce: in the case of FU Ori, dominated by viscous dissipation, the expected temperature exponent is -0.75 . Akeson et al. (2002) separately fit SEDs and visibilities for T Tau and SU Aur but fail to find a set of parameters consistent both with interferometric data and SED.
Table 4. Parameters used for the models of our best fits. For T Tau North the SED and visibility fits are displayed.

\begin{tabular}{lllll}
\hline \hline & SU Aur & FU Ori & $\begin{array}{l}\text { T Tau N } \\
(\mathrm{SED})\end{array}$ & $\begin{array}{l}\text { T Tau N } \\
(V)\end{array}$ \\
\hline$r_{*}\left(R_{\odot}\right)$ & 3.1 & $4.0^{a}$ & 3.5 & $3.8^{d}$ \\
$r_{\min }\left(R_{\odot}\right)$ & 8.2 & 4.4 & 3.6 & $18.0^{d}$ \\
$r_{\max }(\mathrm{AU})$ & $200^{a}$ & $150^{a}$ & $80^{a}$ & $80^{a}$ \\
$M_{*}\left(M_{\odot}\right)$ & 2.2 & 1.0 & 2.0 & $2.0^{d}$ \\
$\dot{M}\left(M_{\odot} / \mathrm{yr}\right)$ & $2.0 \times 10^{-7}$ & $4.0 \times 10^{-5}$ & $1.4 \times 10^{-7} c$ & $8.0 \times 10^{-7} d$ \\
$T_{*}(\mathrm{~K})$ & 5600 & $5000^{a}$ & 4600 & $4700^{d}$ \\
$i\left(^{\mathrm{o}}\right)$ & 40 & 40 & 0 & $0{ }^{d}$ \\
$A_{V}(\mathrm{mag})$ & 0.85 & 1.4 & 0.8 & $0.8^{a}$ \\
$\mathcal{A}$ & 0.3 & 0.2 & $0.3^{c}$ & $0.2^{d}$ \\
$\alpha$ & $2 \times 10^{-4} b$ & $1 \times 10^{-1} b$ & $2 \times 10^{-3} a$ & $1 \times 10^{-2} d$ \\
\hline${ }^{a}$ Parameter not constrained. & & \\
${ }^{b}$ Parameter loosely constrained. \\
${ }^{c}(1-\mathcal{A}) \dot{M}$ constrained. \\
${ }^{d}$ Parameters not separately constrained. \\
\hline
\end{tabular}

Figure 9 displays observational data together with our best models fits.

\subsection{Fitting strategy}

We have identified various resulting characteristics of our modelled SEDs and visibilities that relate more specifically to physical parameters of the model. We describe hereafter how we use them to adjust our fits.

- The level of the near-IR and mid-IR SED are very sensitive to the accretion rate (see Fig. 8d), and more precisely to $M_{*} \dot{M}$ (used to fit $M_{*} \dot{M}$ ).

- The shape of near-IR and mid-IR SED is sensitive to the inner disc truncation (see Fig. 8b) (used to fit $r_{\text {min }}$ ).

- The shape of the optical SED is sensitive to the stellar temperature, the stellar radius and the extinction (used to fit $A_{V}$, $T_{*}$ and $r_{*}$ ). However, in massive discs, the shape of the optical SED is governed by the disc only and the values of $r_{*}$ and $T_{*}$ have little impact on the observables.

- The shape of the far-IR SED is sensitive to the stellar temperature and to the albedo (used to fit $\mathcal{A}$ ).

- The inclination of the disc implies a spread of visibility measurements at a given baseline modulus because the visibilities along major and minor axes then differ. We fit the inclination $i$ using the spread in visibility.

Visibilities have not been used to fit parameters directly but were added as a constraint. The reason is that many parameters have an influence on visibilities, as shown in Fig. 8.

\subsection{SU Aur}

We were able to find a set of parameters to adjust simultaneously the visibilities and the spectrum of this object (see Table 4).

As expected with an accretion rate of $2 \times 10^{-7} M_{\odot} / \mathrm{yr}$, the structure of SU Aur is dominated by viscous heating within 

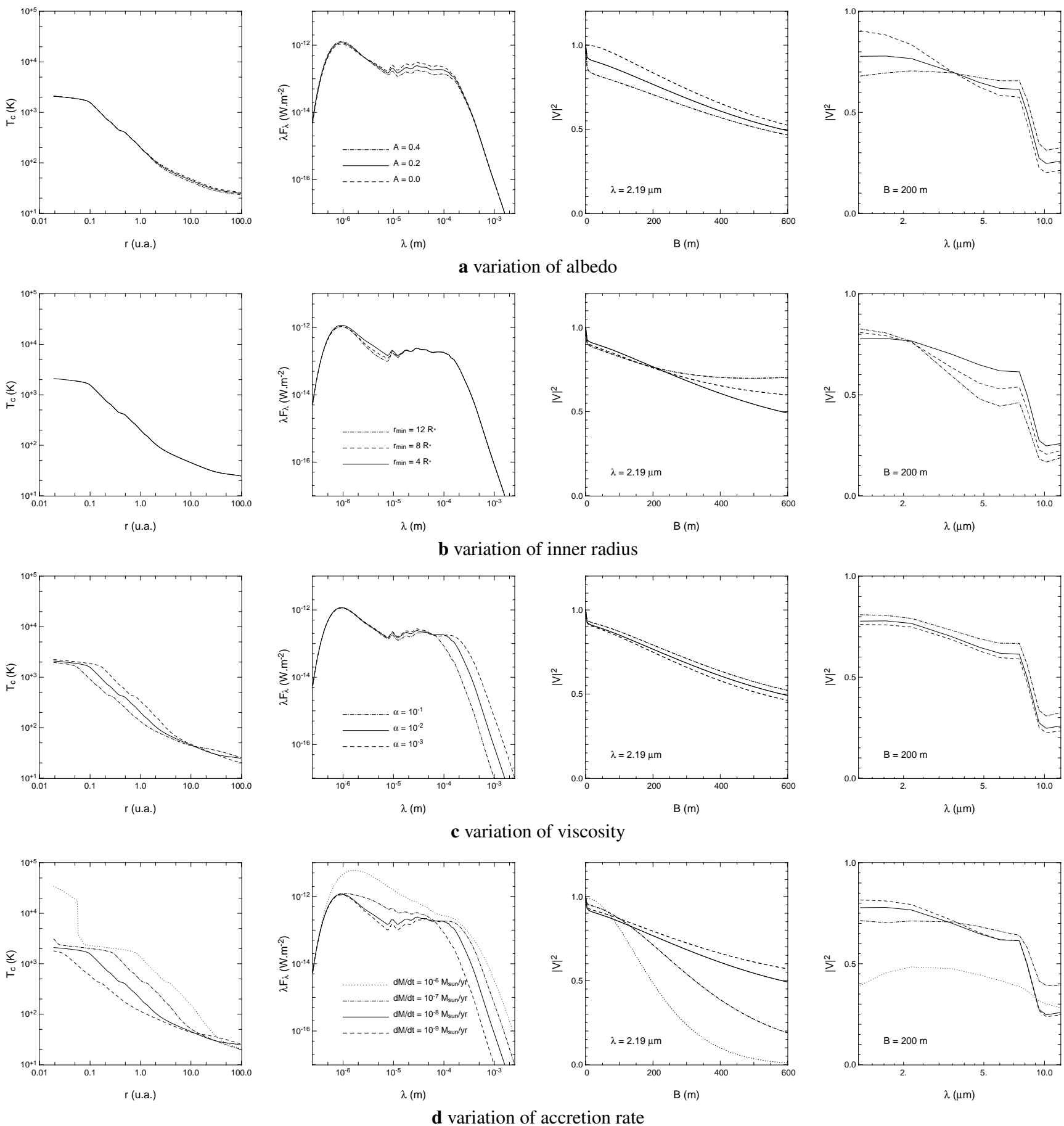

Fig. 8. Variation of the structure of a disc (first column), of its SED (second column), of its visibility curve (third column), and of its visibility as a function of the wavelength (fourth column) when one of its parameters varies.

$10 \mathrm{AU}$ from the star. (See the mid-plane temperature curves on the right panel of Fig. 9a) On the contrary, the effective temperature of the disc is never dominated by viscous dissipation, as shown by effective temperature curves on the same figure. Therefore, the inner parts of the disc can be considered as an active disc in terms of structure but not in terms of observables. The reason for this contradictory behaviour is the following: $T_{\mathrm{v}} \approx T_{\mathrm{r}}$, but since $T_{\mathrm{i}} \approx T_{\mathrm{v}} \tau_{\mathrm{i}}+T_{\mathrm{r}} / 2$ with $\tau_{\mathrm{i}} \gg 1$, we indeed have an inner temperature $T_{\mathrm{i}} \approx T_{\mathrm{v}} \tau_{\mathrm{i}}$, that is dominated by viscous heating.

\subsection{FU Ori}

The visibility measurements present a very large scatter. It may be produced by: (i) a large inclination of the disc (ii) a fully resolved structure. The first hypothesis can be discarded with 

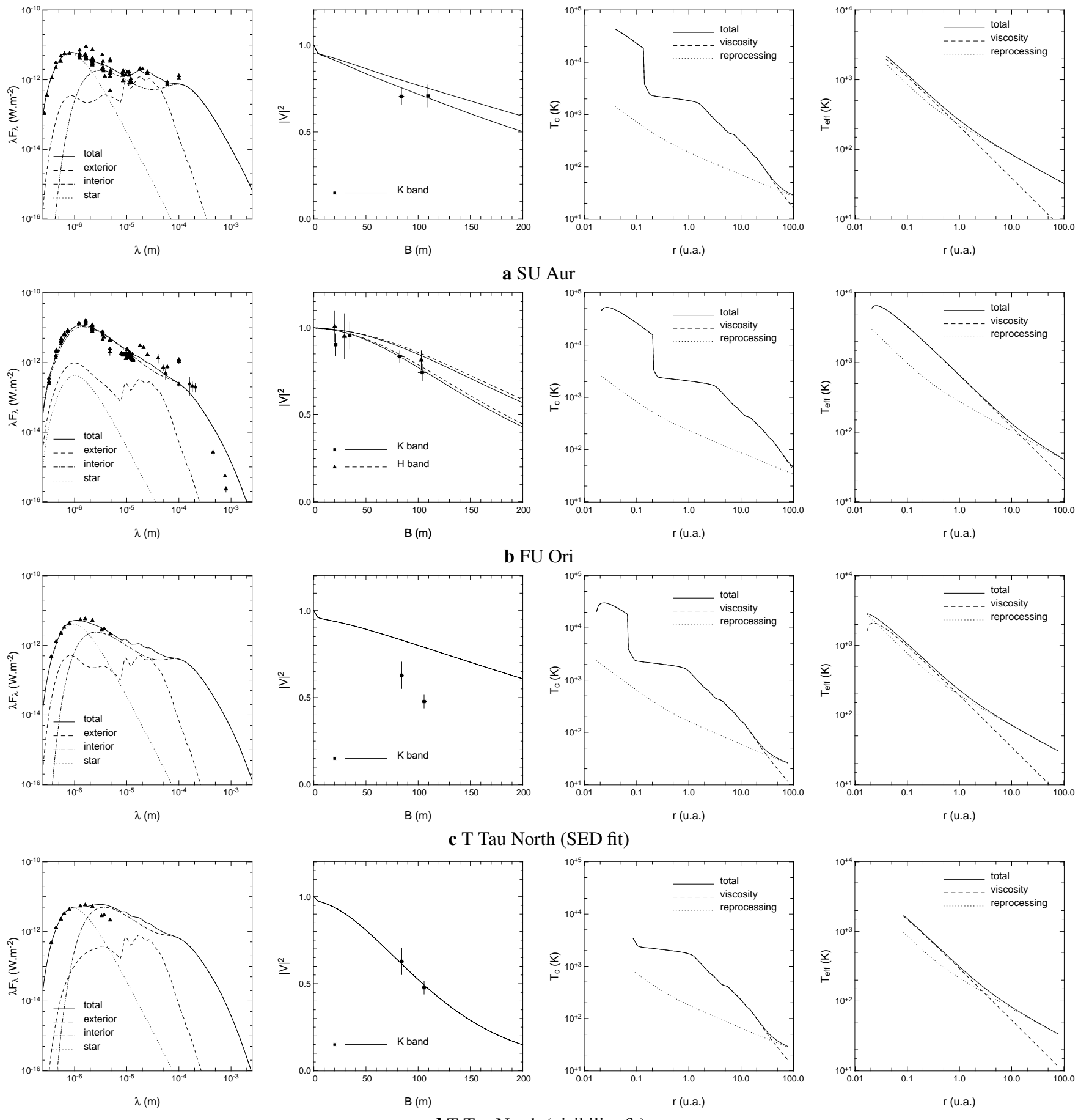

d T Tau North (visibility fit)

Fig. 9. Data and fits for YSOs (a): SU Aur, b): FU Ori, c), d): T Tau North's separate fits for SED and visibility). Left column: SED with contributions of the inner and outer layers and of the star; middle-left column: visibility amplitude vs. baseline, for the major and minor axes of the disc image; middle-right column: mid-plane temperature and contributions of reprocessing and viscous heating vs. radius; right column: effective temperature and contributions of reprocessing and viscous heating vs. radius.

scattered light images that do not show any jet, suggesting that the disc is seen almost pole on. Moreover the low value for a baseline of $30 \mathrm{~m}$ also backs the second hypothesis. Malbet $\&$ Berger (2002) derived that the visibility measurements are compatible with a standard disc if a faint punctual source is located at 35 mas from the star. It might be interpreted as a hot spot in the disc.

We are able to reproduce the SED until $20 \mu \mathrm{m}$ and the average of $H$ and $K$ measurements. The excess in the far IR is a well-know feature likely due to some phenomenon occurring 
in the outer parts of the discs. For instance, Lodato \& Bertin (2001) explain this excess with self-gravitation.

Our model does not explain why the visibility value in the $H$-band at $100 \mathrm{~m}$ is much higher than in the $K$-band while the visibility curve in $H$ is only slightly above the $K$ curve. However our fit is consistent because of large uncertainties on visibility values. We indeed need more accurate measurements to disentangle between uncertainties and model inaccuracy. Malbet \& Berger (2002); Malbet (2002) find that this higher value in $H$ is consistent with an effective temperature distribution $T_{\mathrm{v}} \sim r^{-0.4}$ to -0.6 , that our model cannot reproduce. Figure $9 \mathrm{~b}$ displays the effective temperature of the disc: its law is $T_{\mathrm{v}} \sim r^{-0.75}$, in agreement with the active disc model.

As expected, the structure of FU Ori (see mid-plane temperature curves Fig. 9b, right panel) is dominated by viscous dissipation. Its effective temperature is also dominated by this process within a few tens of AUs from the star.

\subsection{T Tau North}

The available interferometric data for $\mathrm{T}$ Tau North include the $\mathrm{T}$ Tau South component. We have used the Akeson et al. (2002) corrected data to adjust our model but we were unable to derive a unique set of physical parameters to reproduce simultaneously the visibility and the SED of T Tau North. Therefore we present separate fits for the SED and the visibility, respectively displayed in Figs. 9c and 9d. The best SED fit is obtained with a small inner disc truncation and a moderate accretion rate of $2.0 \times 10^{-7} M_{\odot} / \mathrm{yr}$. This fit overestimates the visibility by at least $2 \sigma$. Visibility fits imply a large inner disc truncation and a larger accretion rate, inconsistent with the spectral data.

We cannot exclude caveats in the visibility correction mentioned above. Hence we conclude that the physical parameters derived from the SED fit are the actual T Tau North's ones. Future VLTI or Keck Interferometer (KI) observations with a field of view of 50 mas should remove the T Tau N/S ambiguity.

Like SU Aur, T Tau's disc structure is dominated by viscosity within $10 \mathrm{AU}$ from the star (see Fig. 9c), but in terms of effective temperature both viscosity and reprocessing must be taken into account.

\section{Conclusion}

We have developed an analytical model for $\mathrm{T}$ Tauri accretion discs based on a two-layer approximation, and including the main heating processes: viscous dissipation, reprocessing, and thermalization with the surrounding medium. The outer layer is directly heated by visible stellar light and the thermal flux from the inner layer, whereas the inner layer is heated by viscous dissipation and light reprocessed by the outer layer. The strength of the model is an analytical prediction of the mid-plane temperature and, yet with less accuracy, of the flaring of the disc; it is a suitable tool in grasping the physical conditions taking place in these discs: it allows to predict easily the relative importance of heating processes, the contribution of scattering, the influence of the viscosity prescription, etc.
Despite of simplifications made in order to keep the model analytical, it compares well with other disc models available in the literature. Its predictions in terms of mid-plane temperature, density scale height or disc mass are consistent with those of numerical models by D'Alessio et al. (1998), or by Bell et al. (1997) in the inner regions where this model is valid.

For the first time, we are able to consistently fit the infrared spectra and the optical visibility of two young stars, SU Aur and FU Ori. A third object has been considered (T Tau North), but we could not find a set of parameters that would fit simultaneously the SED and the visibility. This might result from the peculiar structure of this object which is a triple system, and demonstrates that even a single interferometric measurement at one infrared wavelength can be a very strong constraint to interpret disc models. We therefore expect a breakthrough in disc physics understanding when new generation interferometers, like the VLTI or the KI, are able to observe hundreds of young stellar objects. Providing an analytical description and a fast computation, while including essential physical phenomena taking place in discs, our model will be a useful tool to interpret these forthcoming observations.

Concerning the influence of the viscosity on the model output, our results show that current instruments cannot significantly probe the influence of the viscosity law, because it has no direct impact on the flux emerging from optically thick regions. The dependency on viscosity observed in our model remains small and results from the variation of the disc geometrical thickness when the amount of material changes. It is only at larger wavelengths, where the central regions are optically thin, that the mid-plane temperature can be probed, thus indirectly the viscosity. The future Atacama Large Millimetre Array (ALMA) should be able to measure the column density and the mid-plane temperature of discs within 10 AUs from the star, and probe the influence of the viscosity.

In forthcoming work, we will address the following points.

- Including direct heating of the inner rim of the disc by the star will allow us to make predictions for more massive stars (Herbig Ae/Be systems) as shown by Dullemond et al. (2001).

- The treatment of self-shadowing can also be improved: our model 1 assumes that the regions where the disc flares inwards are simply not illuminated at all, while vertical structure models tend to prove that there is still enough material at large heights to catch stellar radiation. Model 2 is neither satisfactory because the flaring index is taken ad hoc, though it gives quite faithful predictions.

- A precise determination of the chemistry species in the outer parts of the disc implies to model the temperature profile in the outer layer instead of assuming an isothermal one. Aikawa et al. (2002) showed that the model by Chiang \& Goldreich (1997) cannot reproduce observed element abundances in the outer part of the disc whereas the vertical structure by D'Alessio et al. (1998) can.

Acknowledgements. We thank the referee Dr. O. Regev for a quick response and a report that helped to clarify the paper. This research has made use of NASA's Astrophysics Data System Bibliographic 
Services, of CDS's Service for Astronomical Catalogues, and of the free software Yorick by D. Munro.

\section{Appendix A: General $\alpha$-disc solution}

In simplified disc models, dealing with optical thickness is always cumbersome, for five different mean optical thicknesses of the inner layer have to be considered: Rosseland optical thickness $\tau_{\mathrm{R}}$, Planck thickness $\tau_{\mathrm{P}}$, Planck-like thickness for the radiation of the outer layer $\tau_{\mathrm{P}}^{e}$ and the Planck-like thickness for the radiation of the star $\tau_{\mathrm{P}}^{*}$. It leads to considering five different regimes summarised in Table A.1. Most T Tauri discs present the regimes 1-4, while the fifth one is seldom encountered. For each of these regimes the mid-plane temperature follows different laws, expressed below:

$T_{\mathrm{i}}^{4} \approx \frac{1}{2} T_{\mathrm{r}}^{4}+T_{\mathrm{v}}^{4} \xi \tau_{\mathrm{R}}+T_{\mathrm{a}}^{4}$,

$T_{\mathrm{i}}^{4} \approx \frac{1}{2} T_{\mathrm{r}}^{4}+T_{\mathrm{v}}^{4}+T_{\mathrm{a}}^{4}$,

$T_{\mathrm{i}}^{4} \approx \frac{1}{2} \frac{1}{2 \tau_{\mathrm{P}}} T_{\mathrm{r}}^{4}+\frac{T_{\mathrm{v}}^{4}}{2 \tau_{\mathrm{P}}}+T_{\mathrm{a}}^{4}$,

$T_{\mathrm{i}}^{4} \approx \frac{1}{2} \frac{\tau_{\mathrm{P}}^{e}}{\tau_{\mathrm{P}}} T_{\mathrm{r}}^{4}+\frac{T_{\mathrm{v}}^{4}}{2 \tau_{\mathrm{P}}}+T_{\mathrm{a}}^{4}$,

$T_{\mathrm{i}}^{4} \approx \frac{1}{2} \frac{\tau_{\mathrm{P}}^{e}}{\tau_{\mathrm{P}}} \frac{2 \tau_{\mathrm{P}}^{*}}{\cos \phi} T_{\mathrm{r}}^{4}+\frac{T_{\mathrm{v}}^{4}}{2 \tau_{\mathrm{P}}}+T_{\mathrm{a}}^{4}$

where $\xi$ is a constant of the order of a few tenths related to the opacity local density exponent $l$ (see Eq. (18)), as defined in Eq. (24).

Some factors 2 in these expressions arise from our choice to deal with half-optical thickness (from mid-plane to outer layer). Instead of using four developments for the first four domains, we use an expression that follows the same asymptotic behaviours:

$T_{\mathrm{i}}^{4}=\frac{T_{\mathrm{v}}^{4}}{F\left(\frac{4}{3 \xi \tau_{\mathrm{R}}}\right) F\left(2 \tau_{\mathrm{P}}\right)}+\frac{T_{\mathrm{r}}^{4} F\left(2 \tau_{\mathrm{P}}^{e}\right)}{2 F\left(2 \tau_{\mathrm{P}}\right)}+T_{\mathrm{a}}^{4}$

with

$F(x)=1-\exp (-x)$

In Eq. (A.2), all F-terms can be interpreted using simple radiative transfer arguments, except for $\frac{4}{3 \xi \tau_{R}}$ that has been taken ad hoc.

Table A.1. Five optical thickness regimes of the disc.

\begin{tabular}{ccccc}
\hline \hline regime & $\tau_{\mathrm{R}}$ & $\tau_{\mathrm{P}}$ & $\tau_{\mathrm{P}}^{e}$ & $\tau_{\mathrm{P}}^{*}$ \\
\hline 1 & $\gg 1$ & $\gg 1$ & $\gg 1$ & $\gg \cos \phi$ \\
2 & $<1$ & $\gg 1$ & $\gg 1$ & $\ll \cos \phi$ \\
3 & $<1$ & $\ll 1$ & $\gg 1$ & $\ll \cos \phi$ \\
4 & $<1$ & $\ll 1$ & $\ll 1$ & $\ll \cos \phi$ \\
5 & $<1$ & $\ll 1$ & $\ll 1$ & $\ll \cos \phi$ \\
\hline
\end{tabular}

The different optical thicknesses of Eq. (A.2) are determined from monochromatic opacities $\kappa_{\lambda}(\rho, T)$ by

$$
\begin{aligned}
& \tau_{\mathrm{R}}=\Sigma \kappa_{\mathrm{R}}\left(\rho, T, T_{\mathrm{e}}\right) \\
& \tau_{\mathrm{P}}=\Sigma \kappa_{\mathrm{P}}(\rho, T, T) \\
& \tau_{\mathrm{P}}^{e}=\Sigma \kappa_{\mathrm{P}}\left(\rho, T, T_{\mathrm{e}}\right)
\end{aligned}
$$

where the generalised Planck and Rosseland opacities of the material at $(\rho, T)$ for black-body radiation at temperature $T_{\mathrm{rad}}$ are given by:

$\kappa_{\mathrm{R}}=\frac{\int_{0}^{+\infty} \frac{\partial B_{\lambda}}{\partial T_{\mathrm{rad}}}\left(T_{\mathrm{rad}}\right) \mathrm{d} \lambda}{\int_{0}^{+\infty} \frac{1}{\kappa_{\lambda}(\rho, T)} \frac{\partial B_{\lambda}}{\partial T_{\mathrm{rad}}}\left(T_{\mathrm{rad}}\right) \mathrm{d} \lambda}$

$\kappa_{\mathrm{P}}=\frac{\int_{0}^{+\infty} \kappa_{\lambda}(\rho, T) B_{\lambda}\left(T_{\mathrm{rad}}\right) \mathrm{d} \lambda}{\int_{0}^{+\infty} B_{\lambda}\left(T_{\mathrm{rad}}\right) \mathrm{d} \lambda}$.

The opacity ratio in the outer layer (thermal radiation/stellar radiation) is then described as

$\omega=\frac{\kappa_{\mathrm{P}}\left(\rho, T_{\mathrm{e}}, T_{\mathrm{e}}\right)}{\kappa_{\mathrm{P}}\left(\rho, T_{\mathrm{e}}, T_{\star}\right)}$.

\section{Appendix B: Flaring index}

The mid-plane temperature is given by

$T_{\mathrm{i}}^{4}=\sum_{k} t_{k}\left(r, T_{\mathrm{i}}\right)^{4}$.

Using Eqs. (10) and (28) one derives

$T_{\mathrm{i}}=\zeta \varepsilon(r)^{2} r$,

where $\zeta$ is a constant and $\varepsilon(r)=\mathcal{H} / r$. Substituting this equation into Eq. (B.1) we have

$\varepsilon(r)^{8}=(\zeta r)^{-4} \sum_{k} t_{k}\left(r, \zeta r \varepsilon(r)^{2}\right)$

The flaring is given by the variation of the relative geometrical thickness $\varepsilon(r)($ Eq. (26)):

$\gamma=\frac{r}{\varepsilon} \frac{\mathrm{d} \varepsilon}{\mathrm{d} r}$.

Derivating Eq. (B.3) in respect to $r$ and introducing

$a_{k}=\frac{\mathrm{d} \log t_{k}^{4}}{\mathrm{~d} \log r}$,

$b_{k}=\frac{\mathrm{d} \log t_{k}^{4}}{\mathrm{~d} \log T_{\mathrm{i}}}$

we derive

$\gamma=\frac{\sum_{k} \gamma_{k} t_{k}\left(r, T_{\mathrm{i}}\right)^{4}}{\sum_{k} t_{k}\left(r, T_{\mathrm{i}}\right)^{4}}$,

with

$\gamma_{k}=\frac{4+a_{k}-b_{k}}{8-2 b_{k}}$.

The flaring index of an optically thick $\alpha$-disc is given in the main matter, by substituting the $t_{k}$ 's in Eq. (B.5). In the following we give the formulae in the case of an arbitrary optical thickness and of a $\beta$-disc. 


\section{B.1. $\alpha$-disc of arbitrary optical thickness}

The expression for the temperature contributions $t_{k}$ are in the present case quite tangled (see Appendix A), though straightforward to derive, so we only give the results for the flaring index. We note

$\eta(x)=\frac{x \exp (-x)}{1-\exp (-x)}$

the logarithmic derivative of $F$. The flaring associated to each heating process are:

$\gamma_{1}=\frac{\mathcal{P}(l, m)\left(\eta\left(2 \tau_{\mathrm{P}}\right)-\eta\left(\frac{4}{3 \xi \tau_{\mathrm{R}}}\right)\right)+(2+g(r))}{Q(l, m)\left(\eta\left(2 \tau_{\mathrm{P}}\right)-\eta\left(\frac{4}{3 \xi \tau_{\mathrm{R}}}\right)\right)+16}$,

$\gamma_{2}=\frac{\mathcal{P}(l, m)\left(\eta\left(2 \tau_{\mathrm{P}}\right)-\eta\left(2 \tau_{\mathrm{P}}^{e}\right)\right)+4}{Q(l, m)\left(\eta\left(2 \tau_{\mathrm{P}}\right)-\eta\left(2 \tau_{\mathrm{P}}^{e}\right)\right)+14}$,

$\gamma_{3}=\frac{\mathcal{P}(l, m)\left(\eta\left(2 \tau_{\mathrm{P}}\right)-\eta\left(2 \tau_{\mathrm{P}}^{e}\right)\right)+2}{Q(l, m)\left(\eta\left(2 \tau_{\mathrm{P}}\right)-\eta\left(2 \tau_{\mathrm{P}}^{e}\right)\right)+16}$,

$\gamma_{4}=\frac{1}{2}$

with

$\mathcal{P}(l, m)=2 m+3 l+1-(1+l) g(r)$,

$Q(l, m)=4 m-6 l-4$.

\section{B.2. Optically thick $\beta$-disc}

The switch to $\beta$-prescription changes terms that involve column density:

$\Sigma_{0}=\frac{f(r) \dot{M}}{3 \pi \beta \Omega r^{2}}$,

$\rho_{0}=\frac{\Sigma_{0}}{r} \sqrt{\frac{2}{\pi}}$.

Then, only the temperature contribution and flaring index related to viscous dissipation are changed:

$$
\begin{aligned}
t_{1}(r)^{4} & =\frac{3}{4} k \mu^{l / 2} T_{\mathrm{g}}^{l / 2} \rho_{0}^{l} \Sigma_{0} T_{\mathrm{v}}^{4} T_{\mathrm{i}}^{m-l / 2}, \\
t_{1}(r)^{4} & \propto f(r)^{l+2} r^{-2 l-7 / 2} T_{\mathrm{i}}^{m-l / 2}, \\
\gamma_{1} & =\frac{1-3 l-2 m}{16+2 l-4 m}+\frac{2+l}{16+2 l-4 m} g(r) .
\end{aligned}
$$

\section{B.3. $\beta$-disc of arbitrary optical thickness}

The flaring indices are given by the same set of equations as in Appendix B.1 except for a change of $Q(l, m)$ :

$$
\begin{aligned}
& \mathcal{P}(l, m)=2 m+3 l+1-(1+l) g(r), \\
& Q(l, m)=4 m-2 l .
\end{aligned}
$$

\section{Appendix C: Disc thickness on the outer edge}

In the main matter, we used an approximate $g_{z} \propto z$ in the determination of the structure of the inner layer, because most of the matter is concentrated within one or two scale heights, with $h_{\mathrm{i}} \ll r$. In determining the vertical location of the disc surface with the same approximation, we noticed a large underestimation of $\mathcal{H}$. The reason is that $\mathcal{H} \approx 4-6 h_{\mathrm{i}}$ is not so small compared to $r$, so that the departure of $g_{z}$ from the linear law cannot be ignored. So, we are bound to considering the exact expression for the gravity field.

In the outer layer, the Planck optical thickness $\tau_{\mathrm{e}}$, the surface density $\Sigma_{\mathrm{e}}$, and the opacity $\kappa_{\mathrm{e}}$ are linked by

$\tau_{\mathrm{e}}=\kappa_{\mathrm{e}} \Sigma_{\mathrm{e}}$

If we use an isothermal layer, $\Sigma_{\mathrm{e}}$ can be substituted by $\rho_{\mathrm{e}} h_{\mathrm{e}}$ where $h_{\mathrm{e}}$ is the scale height of the outer layer and $\rho_{\mathrm{e}}$ the density at its base. $\tau_{\mathrm{e}}$ can be expressed as $\phi_{\mathrm{f}} \omega$, because we expect the incidence $\phi$ of stellar radiation to be dominated by the flaring term. Therefore

$\kappa_{\mathrm{e}} \rho_{\mathrm{e}} h_{\mathrm{e}}=\phi \omega$

To determine $\rho_{\mathrm{e}}$, we solve the vertical hydrostatic equilibrium of the inner layer in the gravity field of the central star

$g_{z}(z)=-\frac{z c_{\mathrm{i}}^{2}}{2 h_{\mathrm{i}}^{2}}\left[1+\left(\frac{z}{r}\right)^{2}\right]^{-3 / 2}$,

which leads to

$\rho(z)=\rho_{\mathrm{i}} \exp \left(-\left(\frac{r}{h}\right)^{2}\left\{1-\left[1+\left(\frac{z}{r}\right)^{2}\right]^{-1 / 2}\right\}\right)$,

with a scale height

$h(z) \propto \sqrt{T(z)\left[1+\left(\frac{Q h_{\mathrm{i}}}{r}\right)^{2}\right]^{-3 / 2}}$.

This allows us to write

$\rho_{\mathrm{e}}=\rho_{\mathrm{i}} \exp \left(-\frac{Q^{2}}{2} \delta_{\rho}\right)$,

$h_{\mathrm{e}}=h_{\mathrm{i}} \sqrt{\frac{T_{\mathrm{e}}}{T_{\mathrm{i}}} \delta_{h}}$,

$\delta_{\rho}$ and $\delta_{h}$ being corrective terms due to the non-linearity of $g(z)$. They are given in Sect. 2.5.3 (Eqs. (36) and (37)). Therefore

$Q=\sqrt{\frac{2}{\delta_{\rho}} \log \left(\frac{\kappa_{\mathrm{e}} \rho_{\mathrm{i}} h_{\mathrm{i}}}{\phi \omega} \sqrt{\frac{T_{\mathrm{e}}}{T_{\mathrm{i}}} \delta_{h}}\right)}$.

Equation (35) in Sect. 2.5.3 is then obtained after the substitution $\phi=\gamma Q h_{\mathrm{i}} / r$. 


\section{References}

Adams, F. C., Lada, C. J., \& Shu, F. H. 1987, ApJ, 312, 788

Aikawa, Y., van Zadelhoff, G. J., van Dishoeck, E. F., \& Herbst, E. 2002, A\&A, 386, 622

Akeson, R. L., Ciardi, D. R., van Belle, G. T., \& Creech-Eakman, M. J. 2002, ApJ, 566, 1124

Akeson, R. L., Ciardi, D. R., van Belle, G. T., Creech-Eakman, M. J., \& Lada, E. A. 2000, ApJ, 543, 313

Bell, K. R., Cassen, P. M., Klahr, H. H., \& Henning, T. 1997, ApJ, 486,372

Bell, K. R., \& Lin, D. N. C. 1994, ApJ, 427, 987

Bertout, C., Basri, G., \& Bouvier, J. 1988, ApJ, 330, 350

Chiang, E. I., \& Goldreich, P. 1997, ApJ, 490, 368

Chiang, E. I., \& Goldreich, P. 1999, ApJ, 519, 279

D’Alessio, P., Calvet, N., Hartmann, L., Lizano, S., \& Cantó, J. 1999, ApJ, 527, 893

D’Alessio, P., Canto, J., Calvet, N., \& Lizano, S. 1998, ApJ, 500, 411
Dullemond, C. P., Dominik, C., \& Natta, A. 2001, ApJ, 560, 957 Gezari, D. Y., Pitts, P. S., \& Schmitz, M. 1999, VizieR Online Data Catalog, 2225, 0

Henning, T., \& Stognienko, R. 1996, A\&A, 311, 291

Hubeny, I. 1990, ApJ, 351, 632

Huré, J.-M., \& Galliano, F. 2001, A\&A, 366, 359

Huré, J.-M., Richard, D., \& Zahn, J.-P. 2001, A\&A, 367, 1087

Kenyon, S. J., \& Hartmann, L. 1987, ApJ, 323, 714

Lodato, G., \& Bertin, G. 2001, A\&A, 375, 455

Lynden-Bell, D., \& Pringle, J. E. 1974, MNRAS, 168, 603

Malbet, F. 2002, in Interferometry for optical astronomy II, Proc. SPIE, 4838, 554

Malbet, F., \& Berger, J.-P. 2002, in SF2A - Scientific Highlights 2001, ed. F. Combes, D. Barret, \& F. Thévenin (EDP Sciences), 457

Malbet, F., Berger, J.-P., Colavita, M. M., et al. 1998, ApJ, 507, 149

Malbet, F., \& Bertout, C. 1991, ApJ, 383, 814

Malbet, F., Lachaume, R., \& Monin, J.-L. 2001, A\&A, 379, 515

Shakura, N. I., \& Sunyaev, R. A. 1973, A\&A, 24, 337 